\title{
VI. Vom Krieg zum Frieden: Wirtschafts- und Sicherheitsprobleme der britischen Außenpolitik nach 1945
}

\section{1945 - Epochenjahr der Geschichte?}

Die bisher aufgezeigten Kontinuitätslinien bestimmen auch die leitenden Fragen für den weiteren Fortgang der Untersuchung. Dabei soll auf dem bisherigen Befund aufgebaut werden, daß der grundlegende Ziel-Mittel-Konflikt der britischen Außen- und Deutschlandpolitik der Zwischenkriegszeit unter dem Einfluß Keynesscher Wirtschafts- und Stabilisierungskonzepte in den britischen Nachkriegsplanungen deutlicher hervortrat und in der Auseinandersetzung mit den Planungsbeiträgen von Keynes sich eine Kontinuität der Faktoren und Ziele abzuzeichnen begann, die den außenpolitischen Entscheidungsprozeß prägten. Das Spannungsmoment ergab sich aus der tendenziellen Unvereinbarkeit des maßgeblich von Keynes beeinflußten Planungsbefunds, daß aus der Appeasement-Politik der Zwischenkriegszeit und dem inneren Reformanspruch auch Konsequenzen hinsichtlich der Grundlagen der britischen Außen- und Deutschlandpolitik zu ziehen waren, und dem ungeachtet wichtiger Ansätze für eine Stabilisierungspolitik gegenüber Deutschland dominanten Primärziel des Machterhalts. Die Akzentuierung der gerade von Keynes herausgestellten strukturellen Bedeutung des Faktors Deutschland für die innere soziale und ökonomische Reformfähigkeit auch über das Ende des Zweiten Weltkrieges hinaus läßt zwangsläufig die Kontinuität der britischen Außenpolitik schärfer hervortreten.

Aus dieser Perspektive stellt sich auch die Frage nach der Tragfähigkeit des insbesondere von der traditionellen Diplomatiegeschichtsschreibung herausgestellten Befunds, das Epochenjahr 1945 markiere einen Bruch in den internationalen Beziehungen. Tatsächlich stechen zahlreiche Elemente der Diskontinuität ins Auge: die Preisgabe isolationistischer Traditionen in der amerikanischen Außenpolitik; die bipolare Ausrichtung der internationalen Beziehungen; der Prozeß der Entkolonisierung; der endgültige Wechsel zum Dollar als maßgeblicher Leitwährung; der Zusammenschluß der westeuropäischen Länder mit dem Ziel eines beschleunigten und effizienteren Wiederaufbaus; schließlich der Wandel der sicherheitspolitischen Grundlagen, den Entwicklung und Aufbau von Nuklearwaffen im verteidigungspolitischen Kalkül der beiden Bündnisse auslösten. „Europa“, so umreißt Andreas Hillgruber den Schwellencharakter des Jahres 1945 , „wurde nun unübersehbar aus einem Hauptträger der Weltpolitik zum Objekt auBereuropäischer Mächte. [...] An die Stelle der überkommenen, wenn auch faktisch bereits seit dem Ersten Weltkrieg überholten europäischen ,Balance-of-Power'-Problematik trat die Auseinandersetzung der beiden Hauptsieger des Zweiten Weltkriegs, der ,Supermächte USA und Sowjetunion. [...] Europa war nur ein, wenn auch bedeutendes Element in ihren weltpolitischen Kalkulationen, Kombinationen und Aktionen in der Nachkriegszeit. Im wesentlichen wurde somit die europäische Geschichte nach $1945 \mathrm{zu}$ einer Geschichte der machtpolitisch und ideologisch einander gegenüberstehenden, den Kontinent politisch spaltenden ,Blöcke 'Ost und West. "1 Soweit Hillgrubers Epochen-

${ }^{1}$ Hillgruber, S. 11. Vgl. auch Mann, S. 21-39, sowie Ruffmann, S. 1289-1298. 
charakterisierung die Realität der Blockkonfrontation nach 1945 in traditionellen Kategorien der Machtpolitik zu fassen versucht, liefert sie eine den Sachverhalt treffende Zustandsbeschreibung. Freilich stellt sich die Frage, ob diese Sicht den Entscheidungsfindungsprozeß der jeweiligen Regierungen vollständig klärt.

Die Vermutung liegt nahe, daß aus der strukturbildenden „Dominanz des Ost-WestKonflikts"2 nach 1945 zugleich die Fragestellungen und das Analyseinstrumentarium des Historikers abgeleitet werden. Dabei richtet sich der Blick zwangsläufig auf das Widerspiel der beiden Mächte USA und Sowjetunion und damit auf die vielfältigen Elemente der Diskontinuität, während die Interessen und Aktionen der den jeweiligen Blöcken zugehörigen Staaten als Funktion dieses Grundkonflikts gesehen werden. Die Übernahme des außenpolitischen Primats als Analyseprinzip durch die Forschung lenkte daher auch bei der Beurteilung der britischen Außen- und Deutschlandpolitik den Blick auf den Kalten Krieg als vorherrschendem Antriebsmoment. Sofern das Foreign Office sich in seinen Entscheidungen tatsächlich vom Kalkül des Machterhalts leiten ließ, können die so ermittelten Befunde sehr wohl Gültigkeit beanspruchen: Der Zäsurcharakter des Jahres 1945, die Konfrontation der Blöcke, ihre Militarisierung und die daraus resultierende Unterordnung der „alten Mächte“ unter die jeweilige Führung zweier rivalisierender Flügelmächte liefern wichtige und unumstößliche Hinweise zur Ermittlung der Antriebskräfte der britischen Außenpolitik nach dem Zweiten Weltkrieg. Freilich bedürfen die aus diesem Blickwinkel getroffenen Aussagen im Einzelfall einer multiperspektivischen Überprüfung. Denn das Interesse an einer Ursachenforschung der Veränderungen in der Weltpolitik, die der Antagonismus der beiden Hauptsiegermächte der Anti-Hitler-Koalition bewirkte, verstellte den Blick für die Frage nach Kontinuität oder Diskontinuität der gewachsenen außenpolitischen Interessenlage und der Entwicklung der innenpolitischen Antriebskräfte von Außenpolitik im Lager der ausscbeidenden Mächte. Im einzelnen bleiben für die Außenpolitik Großbritanniens so entscheidende Faktoren wie etwa die strukturelle Kontinuität von "Appeasement“ in der Deutschlandpolitik der Nachkriegszeit, der langfristige Transformationsprozeß der imperialen bzw. hegemonialen Wirtschaftsstruktur der Pax Britannica Oeconomica in ein Weltwirtschaftssystem mit bipolarer Machtverteilung, der damit verbundene Paradigmenwechsel zu einer keynesianisch orientierten staatlichen Konjunkturpolitik oder auch die seit langem akute Frage nach den innenpolitischen Auswirkungen einer vor allem auf ökonomischem Wege erfolgenden Domestizierung Deutschlands unberücksichtigt.

\section{Kassandras Vermächtnis: Einflußfaktoren der britischen Außenpolitik im Spiegel von Keynes' letzten Aktivitäten}

\section{Bretton Woods als Faktor der britischen Außenpolitik}

Der sichtbare Abwärtstrend in der Entwicklung der britischen Wirtschaft seit der Ankündigung von amerikanischen Lend-Lease-Lieferungen im Januar 1941 und der Verabschiedung des Mutual Aid Act im Februar 1942 führte auch innerhalb der politischen

2 Ritter, S. 89-101; Joffe, S. 25-54; vgl. auch Link. 
Führungsspitze in London zu einem Grundbewußtsein der schwierigen Ausgangsposition, von der aus die künftige britische Außenpolitik der Nachkriegszeit würde starten müssen. Mochten auch die Richtungsvorgaben, die mit den amerikanischen Hilfslieferungen in materieller, mit dem Mutual Aid Act zugleich aber auch in wirtschaftspolitischer Hinsicht angezeigt waren, vorerst nur bei den Experten im Außenministerium und im Schatzamt ein gewisses Krisenbewußtsein schaffen - eine dumpfe Vorahnung, daß die immer stärker werdende Aufbruchstimmung in der Bevölkerung, ihr Gefühl, an einer Zeitenwende zu stehen, und ihre Erwartung, mit dem Ende des entbehrungsreichen Krieges auch die Krisen der Zwischenkriegszeit, die Arbeitslosigkeit und die materiellen Engpässe hinter sich zu lassen, in einem gefährlichen Mißverhältnis zu den tatsächlich vorhandenen wirtschaftlichen Ressourcen stehen würden, war auch in der Entscheidungsspitze anzutreffen.

Bereits zu Beginn des Jahres 1943 warnte Winston Churchill im Vorgefühl des Kommenden vor einem grundlosen und schwer steuerbaren Optimismus. Zwar meldete sich hier nicht zuletzt der unverbesserliche Anti-Keynesianer zu Wort; seine Skepsis brachte jedoch keineswegs grundlose Bedenken auf den Punkt, auch wenn er zu diametral entgegengesetzten deutschlandpolitischen Schlußfolgerungen gelangte. Der allgemeinen, im Beveridge-Plan visionär Ausdruck verliehenen Erwartung, nach dem Krieg würden Arbeitslosigkeit und niedrige Löhne überwunden, die Ausbildungsmöglichkeiten verbessert, der Wohnungsbau und das Gesundheitswesen reformiert, während gleichzeitig die Lebenshaltungskosten nicht steigen und die Spareinlagen der Bevölkerung nicht an Wert verlieren würden, stellte er die düstere Realität der voraussichtlichen außenwirtschaftlichen Lage Großbritanniens entgegen: „Our Foreign Investments have almost disappeared. The United States will be a strong competitor with British shipping. We shall have great difficulties in placing our necessary exports profitably. Meanwhile, in order to help Europe, we are to subject ourselves to a prolonged period of rationing and distribute a large part of our existing stocks. We are to develop the tropical Colonies and raise the condition of their inhabitants. We must keep a large Air Force and Navy so as not to be set upon again by the Germans, and large military forces will be needed to garrison the enemy countries and make sure they do not begin again to re-arm for revenge. The question steals across the mind whether we are not committing our 45 million people to tasks beyond their compass, and laying on them burdens beyond their capacity to bear."3

Diese Mischung aus optimistischer Erwartungshaltung und einer freilich weitgehend unreflektierten Besorgnis hinsichtlich des sich verengenden Spielraums der Außenpolitik wurde zu einem in seiner politischen Antriebswirkung kaum zu überschätzenden mentalen Charakteristikum sowohl in der britischen Öffentlichkeit als auch in Whitehall am Ende des Zweiten Weltkriegs. Wohl war man sich 1945 der kritischen Situation des Landes bewußt, doch verführte der Siegestaumel nur zu leicht dazu, alle dunklen Wolken durch den offensichtlich unbeirrbaren Glauben an einen erfolgreichen, das Sozialprogramm und den Erhalt der britischen Großmachtrolle gleichermaßen garantierenden ökonomischen Wiederaufbau zu vertreiben. Während die Öffentlichkeit aus dem Sieg über Hitler-Deutschland und den durchlittenen Entbehrungen den primär

${ }^{3}$ BLPES, Dalton Papers II, 7/5, fol. 6, Minute Churchill, „Promises About Post-War Conditions“, 10.1.1943. 
moralisch begründeten Anspruch auf soziale Sicherheit und materielle Besserstellung ableitete, herrschte unter den Beamten Whitehalls die Überzeugung vor, daß die Probleme der Übergangszeit vorwiegend technischer und organisatorischer Art sein würden und deshalb grundsätzlich zu bewältigen waren. Der „Optimismus“ entpuppte sich daher bei genauerem Hinsehen als eine nahezu die Form der Gewißheit annehmende Hoffnung, der allerdings noch die harte Prüfung an der Realität bevorstand ${ }^{4}$.

Über diesen Zusammenhang zwischen einem latenten Krisenbewußtsein und dem Glauben an die rasche Überwindbarkeit der weniger strukturell als technisch-organisatorisch bewerteten Probleme des britischen Wiederaufbaus berichtete der amerikanische Regierungsbeamte Lauchlin Currie in einer aufschlußreichen Stimmungsbeschreibung Londoner Regierungskreise am Vorabend der deutschen Kapitulation. Currie, prominentes Mitglied der Foreign Economic Administration des State Department „and one of the ,new deal" braintrusters"5, war im März 1945 nach London zu Gesprächen mit britischen Spitzenbeamten über die wirtschaftliche Lage des Landes und die künftige Behandlung Deutschlands gereist ${ }^{6}$. Die Vermutung liegt nahe, daß Washington mit Blick auf die Zukunft der Lend-Lease-Hilfe sich vor Ort einen Eindruck darüber verschaffen wollte, wie die Entscheidungsspitze die Situation vor dem Wiederaufbau einschätzte. Wie Currie berichtete, zeigten sich die Beamten hinsichtlich der wirtschaftlichen Gesamtperspektive überwiegend zuversichtlich. Als problematisch betrachtete man eher Detailfragen der Übergangsphase von einer Kriegs- zur Friedenswirtschaft. Currie notierte in seinem Bericht weiter, es würde in London weder eine weitverbreitete Arbeitslosigkeit befürchtet, noch sehe man für die britische Zahlungsbilanz langfristig eine Gefahr; man gebe sich sorglos bezüglich der britischen Exportperspektiven nach dem Krieg, und auch für den Wunsch der USA nach einem freien Welthandelssystem habe er prinzipielle Zustimmung verzeichnen können. Auf der anderen Seite berichtete Currie von Skepsis, ob es gelingen werde, die zur Verfügung stehenden Arbeitskräfte in rekonstruktionswirtschaftlich sinnvoller Weise zu verwenden. Dahinter verbarg sich die Furcht, nicht genügend Ressourcen in die Exportwirtschaft lenken zu können, um den lebenswichtigen Ausbau der britischen Außenhandelsposition zu bewerkstelligen.

In gleicher Weise stellte auch Keynes dem US-Ökonomen die Möglichkeit in Aussicht, daß Großbritannien drei Jahre nach der Kapitulation Deutschlands eine ausgeglichene Zahlungsbilanz erreichen könne. Die Chancen, daß dies ohne die Hilfe der USA oder des Internationalen Währungsfonds (IMF) bzw. der Internationalen Bank für Wie-

\footnotetext{
${ }^{4}$ Vgl. aus der zahlreichen Literatur zur innenpolitischen Stimmungslage in Großbritannien in der unmittelbaren Nachkriegszeit Morgan, S. 285-329; Sissons/French (Hrsg.).

${ }^{5}$ BLPES, Meade Papers, Diaries 1/3, fol. 148 (Eintrag 17.3.1945).

${ }^{6}$ FRUS 1945/VI, S. 36-40, Report by Mr. Lauchlin Currie on Conversations with British Officials, March 1945; KCKP, L/E/110-115, Eady an Keynes, 12.3.1945. Currie sprach außer mit Keynes u. a. mit Anthony Eden, Richard Law, Wilfred Eady, David Waley, James Meade und Percivale Liesching. Er lehrte Wirtschaftswissenschaften in Harvard und gehörte bis 1934 dem Finanzministerium an, wechselte dann zum Federal Reserve Board und wurde von Roosevelt zu seinem Administrative Assistant ernannt. Currie gehörte sehr früh zum harten Kern der Keynesianer in den USA und war einer der tatkräftigsten akademischen Trommler für FDRs „New Deal“". Vgl. Galbraith, S. 135, sowie Sweezy, S. $117 \mathrm{f}$. Zu seiner Tätigkeit als Wirtschaftsplaner im Rahmen der amerikanischen Aufrüstungsanstrengungen 1940/41 vgl. Jones, Role, S. 125-133, besonders S. $125 \mathrm{ff}$. Zu Curries Tätigkeit im Zusammenhang mit den amerikanischen Reparationsplanungen im Zweiten Weltkrieg vgl. Nübel, S. 78-84.
} 
deraufbau und Entwicklung gelang, hingen jedoch nach Curries Eindruck von einer Reihe von Bedingungen ab: erstens der Geschwindigkeit, mit der die Exporte aufgebaut würden; zweitens der Dauer des Krieges mit Japan; drittens dem Umfang des britischen Beitrags zu diesem Krieg; und viertens dem Umfang von Lend-Lease während dieser Phase ${ }^{7}$. Mit Keynes' Hinweis auf eine notwendige Übergangsphase zur Erreichung einer ausgeglichenen Zahlungsbilanz deckte sich, was Currie über die handelspolitischen Ansichten Londons nach Washington berichtete. Zwar werde grundsätzlich anerkannt, daß Protektionismus und Bilateralismus als Handelsprinzipien über Bord zu werfen seien. Zugleich werde in Regierungskreisen aber davor gewarnt, Druck auf eine vollständige Revision des bestehenden Systems auszuüben, bevor die britische Wirtschaft ausreichend wiederaufgebaut sein werde. In London sei man der Meinung, so notierte Currie, „that the British commitment to remove exchange restrictions must be conditioned upon a full recovery of equilibrium in the balance of payments; that the principle of imperial preference must remain, although further reductions in the preference might be expected; and that we have probably not given sufficient weight to the strength of protectionist sentiment in Europe and in economically undeveloped countries"8. In London, so lautete die Quintessenz des Currie-Reports, gab man sich also zuversichtlich, sollte es denn gelingen, die vorwiegend technischen Probleme der Übergangsphase, insbesondere die währungs- und handelspolitischen Gespräche mit Washington, die die Lend-Lease-Frage mit einschlossen, sowie die friedenswirtschaftliche Umstellung im britischen Sinne zu lösen. Es war erneut Keynes, der in diesem Zusammenhang gegenüber Currie auch noch einmal seine Besorgnis über den bisherigen Stand der alliierten Planungen zur wirtschaftlichen und militärischen Kontrolle Deutschlands mit Nachdruck vorbrachte. Offensichtlich aufgebracht wegen der grundsätzlichen Entscheidung in Jalta, Deutschland zur Zahlung von Reparationen zu verpflichten, strich er wiederholt die Bedeutung des „First charge“-Prinzips heraus. Keynes betonte, diese Entscheidung liefe angesichts des Zerstörungsgrades der deutschen Industrie und der geplanten Demontage von wichtigen Produktionsanlagen darauf hinaus, daß die USA und Großbritannien Güter nach Deutschland einführen müßten, nur damit die Sowjetunion am anderen Ende Reparationen entnehmen könne. Deutsche Exporte zur Deckung der benötigten Importe, so stellte der Nationalökonom noch einmal unmißverständlich klar, besaßen unbedingten Vorrang vor Reparationen. Es erschien ihm widersinnig, einerseits scharfe Kontrollen über einen längeren Zeitraum aufrechtzuerhalten, nur um andererseits Deutschland zu dem Zweck wieder aufzubauen, um Reparationen abschöpfen zu können. Seiner Meinung nach war es daher sinnvoll, das Land nur so lange zu besetzen, bis die Kriegsverbrecher abgeurteilt und die Rüstungsfirmen demontiert waren, „and then get out, say, in two years' time“"

Currie wußte auch von „einer der kontroversesten, allerdings geheim diskutierten Fragen in britischen Regierungskreisen" zu berichten: ob nämlich Großbritannien bei der Bildung eines westeuropäischen Verteidigungsbündnisses die Führung übernehmen solle. Das Foreign Office, so schilderte er, sei in dieser Frage gespalten. Einige Beamte, wie z. B. der für die Zusammenarbeit mit den Alliierten in Ernährungs- und Rohstoffan-

\footnotetext{
${ }^{7}$ FRUS 1945/VI, S. 37.

${ }^{8}$ FRUS 1945/VI, S. 38.

${ }^{9}$ FRUS 1945/VI, S. 40.
} 
gelegenheiten zuständige Staatssekretär Richard Law und Nigel Ronald, sprachen sich für ein sicherheitspolitisches Engagement auf dem Kontinent aus. Im Mittelpunkt eines Arbeitsessens, an dem neben Currie auch US-Botschafter Winant, dessen Wirtschaftsberater Penrose, Law, Ronald und James Meade teilnahmen, stand die Frage einer westeuropäischen Verteidigungsallianz. Als Currie zu bedenken gab, daß die Schaffung eines Westblocks logischerweise nur gegen die Sowjetunion gerichtet sein könne, erwiderte Law, daß die derzeitige militärische Schwäche Deutschlands ein westeuropäisches Bündnis nicht ausschließe: „Security against whom, if not against Germany?“ Zugleich, so Law weiter, folge daraus keineswegs zwingend, daß die Allianz über kurz oder lang eine antirussische Stoßrichtung einnehmen werde. Sie werde nicht provozierend wirken (und hier schienen alle Beteiligten einer Meinung), wenn erstens die Sowjetunion mit einbezogen oder doch wenigstens um ihre „Zustimmung" zur Bildung der Allianz gebeten würde, zweitens das Bündnis regionaler Teil einer Weltorganisation wäre und drittens eine wirtschaftliche Diskriminierung ausgeschlossen wäre ${ }^{10}$. Demgegenüber bekam Currie von Außenminister Eden zu hören, „that this was not the present policy of the British Government and that he personally is opposed to it. While ostensibly directed against Germany it would actually be directed against the Soviet." 11

Die Hoffnung in London, über die Currie nach Washington berichtete, basierte also auf einer Reihe von miteinander verflochtenen Faktoren, die zum Teil innerhalb der Grenzen des Handlungsspielraums von Großbritannien, zu einem wichtigen Teil aber am Rande oder gar außerhalb seines Einflußbereichs lagen. Zunächst mußte es gelingen, in der Öffentlichkeit Verständnis für eine Politik zur Dämpfung der Nachfrage zu wekken und behutsam auf die Rationierungsmaßnahmen vorzubereiten. In diesem Zusammenhang fürchteten insbesondere die Keynesianer auf beiden Seiten des Atlantiks die Auswirkungen einer amerikanischen Wirtschaftskrise. Zwar hatte Keynes in seinen Überlegungen zur Kriegsfinanzierung genau diese Entwicklung antizipiert und versucht, dem mit seinem Schema des „deferred pay“ ein wirksames Antidot entgegenzustellen. Doch war man in Washington und London skeptisch, ob die Position der Keynesianer genügend gefestigt war, um eine befürchtete wirtschaftliche Depression rechtzeitig einzudämmen, bevor sie die rekonvaleszierenden Staaten Europas mit in den Abgrund riß wie 1929/31'12. Gleichzeitig galt es, namentlich die industrielle Elite von der Notwendigkeit einer grundlegenden Erneuerung der Wirtschaftspolitik zu überzeugen $^{13}$. Schließlich würde es darauf ankommen, in den Verhandlungen mit den USA die

${ }^{10}$ BLPES, Meade Papers, Diaries 1/3, fol. 148-151 (Eintrag 17.3.1945).

${ }^{11}$ FRUS 1945/VI, S. 40.

12 BLPES, Meade Papers, Diaries 1/3, fol. 119-121 (Eintrag 10.2.1945).

${ }^{13}$ Vgl. beispielhaft etwa Keynes' Zorn über den mangelnden Modernisierungswillen der britischen Baumwollindustriellen: "All the cotton leaders", so erklärte er Dalton Anfang 1944, „are ,old jossers who want to live the rest of their lives in peace'. They don't want to be bothered with new ideas or new machinery. The old stuff they are used to will last their time. They are completely defeatist as regards the future and are only thinking of getting back to 1939 . They ought to be thinking of getting back to 1929. Otherwise we are cooked, and can't possibly expand our total exports sufficiently. Who else can supply the post-war world's cotton needs? Germany and Japan will be on their backs, India hasn't our efficiency, the United States costs are too high, Brazil is very small. Who else but we have got the looms and spindles? We shall get good prices for our exports after the war and there is no point in leaving these to be absorbed by foreign merchants." BLPES, Dalton Diaries I/30, fol. 26 (Eintrag 31.1.1944). 
Voraussetzungen für einen stabilen und im Sinne der besonderen britischen Währungsund Handelsinteressen flexiblen organisatorischen Rahmen für den internationalen Zahlungs- und Handelsverkehr zu schaffen ${ }^{14}$. Dieser Aspekt warf nicht nur die Frage nach dem Grad der freihändlerischen Ausrichtung des Systems auf, sondern schloß auch das Problem der Übergangsfinanzierung, genauer: der Kriegsschulden und Kreditmöglichkeiten, mit ein. An seine Seite traten im außenpolitischen bzw. außenwirtschaftspolitischen Bereich die Fragen nach Reparationen, Demontagen und der Art der wirtschaftlichen Kontrolle Deutschlands, die alle die Erfolgsaussichten des britischen Wiederaufbaus auf die eine oder andere Weise beeinflußten.

Alle diese Faktoren nahmen, je näher das Ende des Krieges rückte, immer mehr Keynes' Aufmerksamkeit in Anspruch. Dies um so mehr, als die Arbeitskraft des Nationalökonomen nach Fertigstellung des Malkin-Reports weitgehend von den britischamerikanischen Gesprächen über den Weltwährungsfonds und die Bank für Wiederaufbau und Entwicklung beansprucht wurde. Dies darf jedoch nicht so verstanden werden, als ob er fortan für die deutschlandpolitischen Debatten in Whitehall kein Ohr mehr hatte. Im Gegenteil, wie ausführlich gezeigt wurde, war die umfassende Beschäftigung mit der Frage der wirtschaftlichen Behandlung Deutschlands nach dem Krieg eine zentrale gedankliche Antriebskraft für seine Überlegungen zum ökonomischen Wiederaufbau Europas, so wie vorher seine Kritik an dem Versailler Vertragswerk und die konzeptionelle Konfrontation mit der nationalsozialistischen Wirtschaftsordnung wichtige Impulse für seine theoretische Arbeit geliefert hatten. Namentlich die von ihm im Laufe dieses langen gedanklichen Reifeprozesses diagnostizierte strukturelle Bedeutung des Faktors Deutschland und seiner ökonomischen Dynamik für Großbritannien sorgten dafür, daß Keynes' Interesse an der deutschlandpolitischen Weichenstellung in London auch während seiner umfassenden Aktivitäten bei den Finanzgesprächen in Washington nicht erlahmte.

Keynes' Haltung speiste sich aus zwei auf den ersten Blick widersprüchlichen Antriebsmomenten. Wie kaum ein anderer war er sich der schwierigen wirtschaftlichen Ausgangslage bewußt, der sich Großbritannien am Ende des Krieges gegenübersehen würde; und doch war mindestens ebensosehr der Ökonom in ihm von der Möglichkeit überzeugt, im Inneren die haushalts- und im Äußeren die währungs- und handelspolitischen Grundlagen für einen erfolgreichen Wiederaufbau legen zu können. Während er jedoch hinsichtlich der Möglichkeiten staatlicher Haushaltspolitik als dem Initialzünder für den Wiederaufbau optimistischer als seine Umwelt war, gab er sich im Unterschied zu den Beamten weitaus pessimistischer bei der Beurteilung von Großbritanniens wirtschaftlichen und machtpolitischen Aussichten. Beide Extrempositionen, seine „Micawber-Natur“ wie sein Ruf als „Kassandra“ Whitehalls, waren freilich nur auf den ersten Blick unvereinbar. Denn so überzeugt er davon war, daß er das Instrumentarium für eine Politik der Krisenbewältigung bereitstellen konnte, so sehr verzweifelte er an der Ein-

14 Vgl. dazu z. B. die Hinweise Schatzkanzler Andersons in der Times vom 5.7.1945, in der es u. a. hieß: "The system of international economic collaboration to be established now must profoundly affect our ability to play any useful part in the affairs of the post-war world and may even involve our very standards of life. [...] We must not assume that the cure for all our troubles was found at Bretton Woods. The time is at hand when we must decide and we shall do so heartened immensely by our knowledge of the part which America is clearly determined to play. " $\mathrm{Zi}$ tiert nach: Gardner, Sterling-Dollar Diplomacy, S. 189. 
stellung und den Aktionen weiter Teile aus Industrie und Politik. Aus Keynes' Sicht war es eine Sache, die ökonomischen Warnsignale zur Kenntnis zu nehmen, und eine andere, die richtigen Schlüsse daraus zu ziehen. Eine Politik des „muddle through“ konnte nicht mehr ausreichen, auch nur den Status quo zu erhalten. Um so mehr sah er sich dazu veranlaßt, die Minister die Statistiken nicht nur einfach zur Kenntnis nehmen zu lassen, sondern sie darauf hinzuweisen, daß bei aller Enge des Handlungsspielraums noch politische Entscheidungen gefordert waren, die nicht in hypertropher Verkennung der tatsächlichen Lage an dem Versuch festhielten, die Ressourcen an die Politik anzupassen, sondern die Politik an den wirtschaftlichen Möglichkeiten des Landes auszurichten.

Die Schärfe von Keynes' Kritik wuchs dabei, je näher das Ende des Krieges rückte und je größer seine Unzufriedenheit mit der politischen Entscheidungsspitze wurde. Daneben dürften zunächst freilich zwei weitere Faktoren maßgeblichen Einfluß darauf gehabt haben, daß er gegenüber den Beamten immer häufiger die Dringlichkeit seines Anliegens einforderte. Zum einen kristallisierte sich heraus, daß der in „How to Pay for the War" vorgeschlagene Weg der Kriegsfinanzierung nicht den gewünschten Effekt zeigte. Die im Zuge des Haushalts 1941 eingeleitete Politik steuerlicher „Zwangseinlagen“, die als „deferred pay“ nach dem Krieg für eine ausreichende Gesamtnachfrage sorgen und damit den Grundstock für weitere anti-inflationäre und antizyklische Maßnahmen bilden sollten, blieb weit hinter den Erwartungen zurück ${ }^{15}$. Kurzfristig zwang dies die Regierung dazu, bei den Banken um Kredite nachzusuchen, und durchkreuzte so Keynes' Plan, mit Blick auf die Nachkriegszeit die Bevölkerung und nicht die Kreditinstitute zum Kreditgeber des Staates zu machen. Damit war ein wichtiger Hebel zur Nachfrageregulierung und zugleich ein zentrales soziales Anliegen von Keynes verloren. Zum anderen wuchs der Druck zur Einführung direkter Kontrollen, namentlich im Bereich der Investitionen und zur Dämpfung der Nachfrage, sowie zur Auflösung der Bankreserven; für notwendige Kapitalinvestitionen blieb da kaum Spielraum ${ }^{16}$. Hinsichtlich der Wiederaufbauphase zeichnete sich ab, daß Rationierungen und das kriegsbedingte System der Wirtschaftskontrollen weiter fortbestehen mußten. Hier waren gleichermaßen die Lebensverhältnisse der Bevölkerung, die noch dazu über einen gefährlichen Kaufkraftüberhang verfügen würde, und die Investitionstätigkeit der Industrie direkt betroffen, und es stand zu erwarten, daß dies nicht ohne Auswirkungen auf die erhoffte Steigerung der wirtschaftlichen Produktivität bleiben konnte. Was Keynes zudem in diesem Zusammenhang noch nicht absehen konnte, war der Umstand, daß der allgemein erwartete "Slump“ nach Kriegsende ausblieb, so daß die rückzahlbaren Kredite („post-war credits“) an die Bevölkerung ihren eigentlichen Zweck als entschärfendes Korrektiv in eine potentiell inflationäre Dynamik umkehren konnten ${ }^{17}$.

Darüber hinaus gaben auch die zähen Verhandlungen, die Keynes im unmittelbaren Anschluß an die Fertigstellung des Malkin-Reports mit den Amerikanern im Herbst

15 Vgl. Peden, Keynes, the Treasury and British Economic Policy, S. 42; Harrod, Keynes, S. 494. Vgl. die Angaben bei Pollard, S. 213-216.

16 Vgl. Pollard, S. 214 (Tab. 5.9.) und S. 215.

${ }_{17}$ Zum Problem des Nachfrageüberhangs vor dem Hintergrund allgemeiner Inflationsangst im britischen Schatzamt in der Übergangsphase vgl. Cairncross, Years of Recovery, S. 409-422. Im Zusammenhang mit der Diskussion um die "Keynesianische Revolution“ im Schatzamt vgl. Peden, Keynes, the Treasury and British Economic Policy, S. 42. 
1943 über den Internationalen Währungsfonds führte, Anlaß zu Skepsis ${ }^{18}$. Zwar äußerte sich Keynes über das schließlich im April 1944 veröffentlichte "Joint Statement" ${ }^{19}$, das die beiden von ihm und seinem amerikanischen Kollegen White vorgelegten Pläne zu einem Kompromißpapier zusammenfaßte, vorwiegend positiv. Seiner Ansicht nach war damit durchaus ein erfolgversprechender Grundstein für den wirtschaftlichen Wiederaufbau nach dem Krieg gelegt, und auch im Foreign Office hatte man erkannt, daß, unabhängig von inhaltlichen Details, ein wichtiger, nach außen sichtbarer Schritt getan war, der die USA zwang, Verantwortung für den Wiederaufbau und die Sicherheit Europas zu übernehmen ${ }^{20}$. Freilich war Keynes gezwungen, den auf der Konferenz von Bretton Woods im Juli 1944 bestätigten Kompromiß einer skeptischen Öffentlichkeit in Großbritannien, die um die wirtschaftspolitische Entscheidungsfreiheit der Regierung bei der Erhaltung britischer Interessen besorgt war, und namentlich den einer multilateralen Öffnung des Landes ablehnend gegenüberstehenden zuständigen parlamentarischen Instanzen zu verkaufen ${ }^{21}$. Bei allen Vorbehalten gegenüber dem amerikanischen Plan sah Keynes doch zu den Prinzipien von Multilateralität und Währungskonvertibilität keine prinzipielle Alternative, und seine Vorschläge zur wirtschaftlichen Neuordnung gründeten im Kern ebenso auf dem Grundsatz der internationalen Zusammenarbeit wie die Washingtons. Bei einer Ablehnung durch die Parlamente drohte allerdings der Rückfall in protektionistische Extrempositionen und damit ein kaum wiedergutzumachender Schaden in den Beziehungen zu Washington, auf dessen Hilfsbereitschaft Großbritannien gerade im Hinblick auf die Finanzierung der Übergangsperiode angewiesen sein würde ${ }^{22}$. Doch obwohl Keynes zentrale Aspekte seiner eigenen Vorlage in das "Joint Statement“ einzubringen vermochte und obwohl es ihm gelang, wichtige „Rückzugsklauseln“ einzubauen, die Großbritannien ein rechtzeitiges Gegensteuern für den Fall erlauben sollten, daß der Druck auf die Währung zu groß wurde, war er sich nur zu bewußt, daß es ihm grundsätzlich nicht gelungen war, die seinen eigenen Clearing UnionPlänen unterliegenden praktischen Zielvorgaben durchzusetzen und allgemein die amerikanischen Verhandlungspartner von der speziellen Situation Großbritanniens zu überzeugen. Denn so erfreulich die prinzipiellen Berührungspunkte der britischen und amerikanischen Pläne auch waren, so fundamental unterschieden sich ihre Interessen in der Frage, welche Weichenstellungen man mittelfristig für die Übergangsphase vornehmen sollte.

Die von Keynes bei den Washingtoner Verhandlungen im September und Oktober 1943 verfolgten Ziele können wie folgt umrissen werden ${ }^{23}$ :

18 Vgl. Moggridge, Maynard Keynes, S. 775-780.

${ }^{19}$ Vgl. Joint Statement by Experts on the Establisment of an International Monetary Fund, London 1944 (Cmd 6519).

${ }^{20}$ Memorandum Hall-Patch, 3.8.1945, in: DBPO I/3, S. 1-5, besonders S. 4.

21 Vgl. ausführlich Gardner, Sterling-Dollar Diplomacy, S. 121-129.

22 Zur Reaktion in Großbritannien auf das "Joint Statement" vgl. Gardner, Sterling-Dollar Diplomacy, S. 121-129; zu Keynes' Überzeugungsarbeit ebenda, S. 124-129; vgl. auch Harrod, Keynes, S. 569-575, 581-584.

${ }^{23}$ Aus der Fülle der Literatur zum Bretton Woods-Abkommen sei hier beispielhaft verwiesen auf Gardner, Sterling-Dollar Diplomacy, S. 71-100, 110-144; van Dormael. Aus Keynes' Sicht Harrod, Keynes, S. 555-585; Moggridge, Maynard Keynes, S. 721-755. Aus der Sicht zweier Beteiligter White sowie Meade, Bretton. Woods. Grundsätzlich sei verwiesen auf das Material in JMK XXVI. 
- Nach dem Willen Londons sollte der IMF zur wichtigsten Nachkriegsquelle für weitgehend konditionslose Kredite aufgebaut werden. Aus diesem Grunde sah Keynes' Clearing Union-Entwurf Kreditmöglichkeiten der Mitgliedsländer in Höhe von \$26 Milliarden vor, die zu fast $90 \%$ von den USA als größtem Kreditgeber eingebracht werden sollten.

- Die britische Verhandlungsdelegation zielte zugleich auf eine Reduzierung bzw. Eliminierung amerikanischer Zölle, um so langfristig den Zugang zu den Dollarmärkten zu garantieren und damit für die kriegsgeschädigten Volkswirtschaften die Möglichkeit des Devisenerwerbs zu schaffen. Zu diesem Zweck strebten die britischen Unterhändler eine Formel an, die eine automatische Reduzierung der Zollbestimmungen der Mitgliedländer festschrieb. In den Augen Londons war dies eine Grundvoraussetzung für den Artikel VII des Mutual Aid Act, der die Prinzipien des Multilateralismus verpflichtend vorschrieb. Das System der Empire-Präferenzen war nur in dem Maße preiszugeben, wie die Amerikaner zur Lockerung ihrer Importbeschränkungen bereit waren.

- Dem Doppelziel, monetäre Rahmenbedingungen zu schaffen, die die Einnahme von Dollars erleichterten, ohne zugleich den britischen Anspruch auf fiskalische Expansion im Inneren aufgeben zu müssen, diente auch die sogenannte „scarce currency

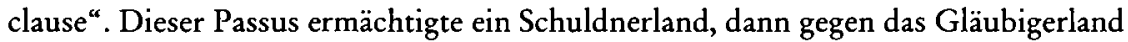
diskriminierend vorzugehen, wenn dessen positive Zahlungsbilanz eine bestimmte Grenze überschritt und (Dollar-)Exporte der Schuldnerländer dadurch so erschwert wurden, daß die Reserven des IMF in dieser Währung zu verknappen drohten ${ }^{24}$. Dadurch sollte verhindert werden, daß eine allgemeine Devisenknappheit, die durch kriegsbedingte Überkapazitäten der amerikanischen Wirtschaft und konstant hohe Importzölle noch verschärft zu werden drohte, den Fluß des Welthandels und damit den allgemeinen Wiederaufbau behinderte. Daneben hoffte Keynes, London die Möglichkeit der Währungskontrolle, insbesondere die Option der Abwertung vom Sterling, als eine Maßnahme zur Absicherung der Reformen im Inneren offenzuhalten.

- In dem Maße, wie sich herausstellte, daß die britische Delegation die Details der amerikanischen Vorlage weitgehend zu akzeptieren gezwungen war und die Amerikaner sich insbesondere weigerten, mit dem IMF einen allgemeinen Dollarpool in der von Keynes anvisierten Größenordnung zu schaffen, mußte es darauf ankommen, nach Ausgleichsmechanismen zu suchen; die Verhandlungen dienten Keynes also zugleich dazu, den Grund für bilaterale Hilfsmaßnahmen zwischen den USA und Großbritannien zu legen.

Auch wenn die britische Delegation am Ende der Verhandlungen Teilerfolge verbuchen konnte und die USA prinzipiell an den europäischen Wiederaufbau im Rahmen einer Konsolidierung des Weltwährungssystems anbinden konnte (ein Ergebnis, das den eigentlichen Stellenwert von Bretton Woods kennzeichnet), kann doch kaum ein Zweifel darüber bestehen, daß weder die Ergebnisse der Finanz-noch die der Handelsgespräche die Rahmenbedingungen schufen, die man sich in London im Hinblick auf eine rasche Konsolidierung versprochen hatte. So gelang es den Amerikanern nicht nur, unter Hin-

${ }^{24}$ Vgl. dazu ausführlich Harrod, Keynes, S. 543-548; Gardner, Sterling-Dollar Diplomacy, S. 91 f., 114-117, 125-127, 137. 
weis auf den widerspenstigen Kongreß alle britischen Versuche abzuwehren, die automatische Reduzierung der Zollbeschränkungen für die Mitgliedsländer festzuschreiben; sie sahen sich überdies auch keineswegs veranlaßt, im Gegenzug ihren Druck auf Abbau der Empire-Präferenzen zu reduzieren ${ }^{25}$. Zudem weigerten sie sich erfolgreich, dem Währungsfonds die von Keynes geforderte finanzielle Basis von \$26 Milliarden zu garantieren, d. h. einen Eigenanteil von \$23 Milliarden aufzubringen. Damit war der Kern von Keynes' Plan, den IMF mit einer ausreichenden Menge der amerikanischen Währung auszustatten, über die die Mitgliedsländer konditionslos verfügen konnten, zerstört. Das Problem des Devisenengpasses in der Übergangsphase, und damit das Kernproblem der britischen Zahlungsbilanz, schwelte also weiter. Ob die ins Abkommen geschriebene „scarce currency clause“ als "Trostpreis" 26 für die Zugeständnisse der Briten genügend währungspolitischen Spielraum bot, durfte bezweifelt werden, zumal Washington auf zahlreichen, die Anwendung der Klausel einschränkenden Passagen bestand ${ }^{27}$. Bestenfalls konnte sie dazu dienen, im Notfall den Druck auf die eigenen Devisenreserven einzudämmen; ein Mechanismus, mit dem sich mehr Dollars erwirtschaften ließen, war sie gewiß nicht. Und schließlich standen am Ende der Bretton Woods-Konferenz berechtigte Zweifel, ob die USA bereit sein würden, Großbritannien Sonderkonditionen im Rahmen von Hilfsmaßnahmen nach dem Ende von Lend-Lease einzuräumen. Jedenfalls waren die Anhörungen in Senat und Repräsentantenhaus ein schlechtes Omen; dort vertrat man diesbezüglich die Meinung, „that if Bretton Woods agreements are adopted, there will be no direct aid [for Great Britain] - at any rate not in the form of lend-lease or in the form of a credit on terms which England could afford " 28 . Die engere währungspolitische Anbindung der USA an Europa durch das Bretton-Woods-System legitimierte sich in den Augen der amerikanischen Öffentlichkeit gerade dadurch, daß sie den Steuerzahler von jeder weiteren Belastung durch Hilfsgelder befreien sollte.

\section{Amerikanische Finanzhilfe nach dem Krieg? Keynes' Lageanalyse vom August 1944}

Die Veröffentlichung des "Joint Statement" markiert einen bedeutenden Einschnitt in den Nachkriegsplanungen Großbritanniens nicht nur, weil diese Erklärung wichtige Grenzpfähle des außenpolitischen Handlungsspielraums markierte; seine Veröffentlichung im April 1944 fiel vielmehr auch zusammen mit der an anderer Stelle skizzierten Reorganisation der Planungsstäbe in London, namentlich mit der Schaffung des EIPS im gleichen Monat, der wenig später mit der Ausarbeitung einer Studie über „Economic Security" begann".

Im Juni dieses Jahres, kurz vor der Konferenz von Bretton Woods, legte Keynes eine erste Denkschrift zu Fragen der britischen Außenwirtschaft in der Übergangsphase

\footnotetext{
${ }^{25}$ Vgl. im einzelnen Gardner, Sterling-Dollar Diplomacy, S. 150-153.

${ }^{26}$ Harrod, Keynes, S. 548.

${ }^{27}$ Vgl. Meade, Bretton Woods, S. 9 f.; Gardner, Sterling-Dollar Diplomacy, S. $116 \mathrm{f}$.

${ }^{28}$ So resümierend John $\mathrm{H}$. Williams, Wirtschaftswissenschaftler in Harvard und Vizepräsident der Federal Reserve Bank in New York; zitiert nach: Gardner, Sterling-Dollar Diplomacy, S. 144; vgl. insgesamt ebenda, S. 129-144.

29 Vgl. oben Kap. IV.1.
} 
vor $^{30}$. Man darf annehmen, daß sie unter dem Eindruck sowohl der Ergebnisse der britisch-amerikanischen Finanzgespräche wie auch der Entwicklung der deutschlandpolitischen Debatte entstand. Keynes lenkte darin die Aufmerksamkeit auf die gefährliche Verschuldung des Landes und zeichnete ein düsteres Bild des britischen Wiederaufbaus. Da war zum einen der kriegsbedingte dramatische Rückgang der britischen Exporte, die die dringend benötigten Einfuhren schon lange nicht mehr deckten; als Folge hiervon wurde die Zahlungsbilanz mit den Ländern außerhalb des Sterling-Blocks in die roten Zahlen gedrückt. Zum anderen sah sich die Regierung mit einer beispiellosen Verschuldung gegenüber den Sterling-Ländern insgesamt konfrontiert. Die hatten nämlich ihre Devisen zentral in London gelagert, so daß Großbritannien darüber verfügen konnte, akkumulierten auf diese Weise aber beträchtliche Beträge in Sterling auf ihren Konten. Um den Schuldenberg abzutragen, überließ ihnen die Regierung in London im Laufe des Krieges immer größere Teile ihrer Investitionsanlagen in Übersee - und setzte damit jene Spirale in Gang, die, stärker als im Ersten Weltkrieg, als Großbritannien nicht gezwungen war, Auslandsinvestitionen in solchem Umfang zu liquidieren, zu einer krisenhaften Zuspitzung der Währungssituation führte. Doch trotz des Abbaus von Überseeinvestitionen blieben nach Keynes' Schätzungen fast $£ 3$ Milliarden auf den Londoner Konten; und dieser Sterlingüberhang war nichts anderes als ein langfristig angelegter Sprengsatz, der dann zünden würde, wenn es nicht gelang, ihn vor Ablauf der im Abkommen von Bretton Woods garantierten Übergangsphase und vor dem Beginn der Konvertibilität des Pfundes ausreichend abzubauen.

Keynes scheute sich nicht, in diesem Zusammenhang auf die leidige britische Tradition des "muddle through" hinzuweisen und die Minister davor zu warnen, daß eine mangelnde Bereitschaft zum Umdenken und eine geistige Inflexibilität gegenüber neuen Entwicklungen noch mehr als nach dem Ersten Weltkrieg bestraft würden. „There has never been a more distinguished example of ,It will all come right on the day “", hielt er den Ministern vor. Im Detail bekräftigte er die Notwendigkeit, zur Überwindung der katastrophalen Devisenlage des Landes um spezielle Hilfe bei den Amerikanern nachzusuchen. Die Fortschreibung von Lend-Lease über das Kriegsende hinaus sollte erstens den Ressourcendruck mindern, um auf diese Weise den Spielraum zur Erwirtschaftung von Gold- und Dollarreserven zu erweitern. Zweitens waren zu diesem Zweck alle verfügbaren Kräfte in den Ausbau der Exportindustrie zu stecken. Drittens war durch Verhandlungen mit den Sterling-Ländern sicherzustellen, daß deren Verfügungsgewalt über die Sterlingguthaben unter Kontrolle und die währungs- und handelspolitische Geschlossenheit des Empire-Blocks unangetastet blieb. Neben diesen im engeren Sinne wirtschaftspolitischen Maßnahmen fügte Keynes schließlich die Forderung hinzu, daß alle außenpolitischen Verpflichtungen, welche die Möglichkeiten des Landes überstiegen, zu vermeiden seien. Auch in der Diplomatie, so seine Schlußfolgerung, waren Neuorientierungen sowie strukturelle Anpassungsmaßnahmen unerläßlich, wenn die Appeasementtendenzen in der britischen Außenpolitik überwunden und ausreichende Ressourcen für den sozio-ökonomischen Wiederaufbau geschaffen werden sollten. In

${ }^{30}$ CAB 66/52, W.P. (44) 360, Annex, Memorandum Keynes, "The Problem of Our External Finance in the Transition“, 12.6.1944; JMK XXIV, S. 34-65; dort auch die folgenden Zitate (Hervorhebungen im Original). Vgl. auch Harrod, Keynes, S. 574f.; Moggridge, Maynard Keynes, S. 762765. 
einer ebenso prägnanten wie stilistisch großartigen Analyse ging der Ökonom mit dem Großmachtanspruch des Landes und seiner Appeasementtradition ins Gericht: „Our present attitude towards our Allies and Associates is the result of several ingredients. In the financial field we have never escaped from the consequences of the Dunkirk atmosphere, when we felt alone: that this is our war; that if anyone helps it is very nice of them, but we cannot, of course, expect that it should be otherwise than on their own terms; that so far as we, but not they, are concerned, the future must be entirely sacrificed to the overwhelming needs of the present; and that if anyone wants a douceur he must, in the interests of getting on with the war, have it. This is the ingredient of appeasement, right and inevitable once, not so clearly necessary now. Next there is our position as a Great Power, equal in authority and responsibility and therefore equal in the assumption of burdens. This is the ingredient of pride and prestige - easily understandable, but nevertheless shortsighted if pride and prestige are, in fact, to be preserved. And, finally, the most sympathetic and natural of all the ingredients, what we have called the gracious activities of Lady Bountiful, all-oblivious of the bailiff's clutch, the universal and unthinking benevolence of a family which has always felt rich and for whom charity has become not so much a sacrifice as a convention."

Indirekte Bestätigung erhielten die Ausführungen von Keynes durch eine Kabinettsvorlage des Schatzkanzlers über die internen Finanzbelastungen ${ }^{31}$. Anderson erläuterte ausführlich die enormen Kosten der vorgeschlagenen Sozialpläne, die eine Erhöhung der Staatsausgaben erforderten, „appreciably bigger than that involved in any other of the post-war commitments we are considering“. Im einzelnen kämen auf Steuerzahler, Unternehmer und Arbeiter beträchtliche zusätzliche Abgaben zu; es bleibe abzuwarten, ob die Bevölkerung, von der ein großer Teil sogar mehrfach betroffen sein werde, dies mitzutragen bereit sein werde ${ }^{32}$. Darüber hinaus sah Anderson zahlreiche weitere Belastungen des Staatssäckels voraus:

- Zinsen für die im Krieg akkumulierten Staatsschulden;

- neben dem erwähnten Sozialversicherungsplan zusätzliche Verpflichtungen im Sozialbereich, etwa freie Schulverpflegung, Kriegsrenten, Wohnungsbau und die Beseitigung von Kriegsschäden;

- Unterstützung der Industrie im Rahmen umfassender Modernisierungs- und Wiederaufbaumaßnahmen;

- Kosten für den Unterhalt der Streitkräfte nach dem Krieg;

- schließlich die Reduzierung der an die Ausnahmesituation des Krieges angepaßten Steuerraten sowie die steuerliche Rückerstattung im Rahmen der „deferred pay“-Politik.

Die große Unbekannte auf der Ausgabenseite, so erläuterte Anderson in Antizipation der tatsächlichen Krisenentwicklung der Nachkriegsjahre, waren die voraussichtlichen

${ }^{31} \mathrm{CAB}$ 66/52, W.P. (44) 353, Memorandum by the Chancellor of the Exchequer, „Post-War Financial Commitments", 28.6.1944; dort auch die folgenden Zitate.

32 Einige Zahlen: Die Sozialpläne sahen für 1945 eine Erhöhung des Arbeitgeberanteils an den Sozialversicherungsbeiträgen von gegenwärtig $£ 87$ Mio. auf insgesamt $£ 124$ Mio. vor; dies entsprach fast einer Verdoppelung des Vorkriegsaufwands (1938/39: $£ 66$ Mio.). Der Mehrbetrag für die Arbeitnehmer belief sich auf voraussichtlich $£ 109$ Mio. und stieg auf insgesamt $£ 178$ Mio. (gegenüber $£ 55$ Mio. 1938/39). Hinzu kam eine weitere Mehrbelastung des Steuerzahlers in Höhe von $£ 78$ Mio. Alle Angaben ebenda. 
Verteidigungsausgaben. Die Schätzungen des Schatzamts beliefen sich, in dramatischer Verkennung der umfassenden militärischen Verpflichtungen des Landes nach dem Krieg, auf etwa $£ 500$ Mio. jährlich. Das Dilemma sei, daß man wohl nicht so lange mit Steuersenkungen warten könne, bis die internationale Situation eine allgemeine Abrüstung zulasse. Wie weit die Schere zwischen Steueraufkommen und Rüstungslasten im Laufe der Zeit auseinanderklaffen und wie die entstehende Lücke zu schließen sein würde, ließ der Schatzkanzler freilich offen. Seine Skepsis konnte er jedoch auch nicht verhehlen: Während etwa der Schuldenberg nach den Napoleonischen Kriegen erträglich gewesen sei, weil eine expandierende Weltwirtschaft mit Großbritannien als industriellem und finanziellem Zentrum im 19. Jahrhundert für die notwendigen Mehreinnahmen gesorgt habe, und auch nach dem Ersten Weltkrieg durch Einkommens- und Arbeitskräftezuwachs die steuerlichen Einnahmen weiter gestiegen seien, müsse jetzt bezweifelt werden, ob das Weltwirtschaftssystem dieses Mal solche expansiven Züge aufweisen werde. Und selbst dann bestanden für Anderson erhebliche Zweifel, ob Großbritannien überhaupt in der Lage sein würde, sich seinen Anteil an einem erneuten Wachstumsprozeß zu sichern - eine vor dem Hintergrund des tatsächlichen britischen Anteils an dem beispiellosen Wachstumsprozeß der fünfziger und sechziger Jahre geradezu beklemmende Weitsicht. Anderson machte deutlich, daß unter diesen Bedingungen der auch in Zukunft wachsende finanzielle Aufwand, den die Sozialpolitik sowie die Maßnahmen für den industriellen und wirtschaftlichen Wiederaufbau notwendig machten, eine ernsthafte Belastung darstellte; umgekehrt durfte man sich nur wenig strukturelle Entlastung versprechen, denn „any serious reduction in the cost of the Armed Forces is a matter for hope rather than for confidence". Am Ende zog der Schatzkanzler die kaum ermutigende Bilanz, es sei unerläßlich, „to appreciate the long-term financial problem in its true perspective, since otherwise sub-conscious beliefs, based on a past experience that is no longer relevant, may create a misleading impression that time is bound to relieve our financial difficulties. It is especially important to scrutinise very carefully all proposed commitments that are likely to entail automatic increases of expenditure. [...] We are, I think, compelled to look at our existing and prospective commitments from the point of view of priorities."

Für das Foreign Office kommentierte dessen Erster Sekretär, J. E. Coulson, Keynes' Ausführungen. Der enge finanzielle Rahmen, so bemerkte er zutreffend, bedeute eine ernsthafte Belastung für die britische Diplomatie. „Not only shall we be much handicapped by lack of money for carrying out our foreign policy, but we shall also be involved in highly delicate negotiations connected with financial matters which will require the utmost attention by the Foreign Office." Wenn man unter diesen Bedingungen mit einer wünschenswerten Außenpolitik Schritt halten wolle, sei es unbedingt notwendig, daß die betroffenen Ministerien in engem Kontakt zum Außenministerium stünden ${ }^{33}$.

Die Meinung im Kabinett zu den von Keynes aufgeworfenen Fragen gingen freilich auseinander. Auf der einen Seite erklärten Konservative wie der Pressezar Lord Beaverbrook, ein einflußreicher Churchill-Intimus und zu diesem Zeitpunkt Lord Privy Seal, und Leo Amery, Secretary of State for India, die Ausführungen von Keynes seien zu pessimistisch, da der Schuldenberg im Kern ein Sterlingdefizit und dementsprechend leicht innerhalb der Empire-Bilanzen auszugleichen sei; überdies, so fügten sie verharmlosend

${ }^{33}$ FO 371/40952/UE 169, Minute J. E. Coulson, 18.7.1944. 
hinzu, könne der US-Dollar genauso schnell wie Sterling einen Kursverlust erleiden. Beaverbrook hielt es deshalb auch für „äußerst bedauerlich“, sollte Keynes in den USA auf die schwierige Finanzlage Großbritanniens hingewiesen haben, und warnte das Kabinett vor in seinen Augen ungerechtfertigten Schwächebekundungen, die den Machtanspruch des Landes von vorneherein unterminieren könnten ${ }^{34}$.

Demgegenüber erklärten sich andere Minister mit der Keynesschen Analyse einverstanden, setzten allerdings unterschiedliche Akzente. So sah Arbeitsminister Bevin den wirtschaftspolitischen Primat in der Entwicklung des Binnenmarktes, fügte jedoch hinzu, daß ein angemessener Anteil der industriellen Produktionskapazität für den AuBenhandel reserviert werden müsse ${ }^{35}$. Schatzkanzler Anderson stimmte angesichts des Befundes seiner eigenen Denkschrift mit dem generellen Tenor von Keynes' Aufzeichnung überein, mahnte aber ebenfalls zur Vorsicht in Verhandlungen mit Washington. Zwar war im Schatzamt allgemein die Notwendigkeit amerikanischer Finanzhilfe unbestritten. Anderson schwebte allerdings - in sentimentaler Erinnerung an die Atlantikschlacht - eher eine britisch-amerikanische Wirtschaftskooperation hinsichtlich des europäischen Wiederaufbaus vor ${ }^{36}$. Was die eigentliche Finanzhilfe anging, sollte sie nach Möglichkeit nur einen nachgeordneten Rang einnehmen. Demgegenüber war er wie Bevin der Meinung, daß die Maßnahmen zur Entlastung des wirtschaftlichen Drucks primär im Bereich der Innenpolitik zu suchen seien: „if we are to avoid financial embarrassment after the war which would not only restrict our power to fulfil some of our international obligations but would confine our freedom of policy and might hinder the development of our internal plans, we shall have to tackle the problem, as we have tackled war production, continuously and over a wide field. " ${ }^{37}$ Dies war nichts anderes als die Fortschreibung der Wirtschaftskontrollen nach dem Krieg, um die Abhängigkeit von amerikanischer Hilfe so niedrig wie möglich zu halten.

Unter dem Strich wurde so auch bei jenen, die Keynes' Analyse im Prinzip zustimmten, der Wille deutlich, die wirtschaftspolitische Entscheidungsfreiheit Londons nicht zuletzt wegen ihrer gesamtpolitischen Bedeutung unter allen Umständen zu verteidigen. In diesem Sinne beschloß das Kabinett schließlich, erstens das Terrain für Verhandlungen mit Washington über den möglichen Umfang der amerikanischen Finanzhilfe nach der deutschen Kapitulation („Stage II“) zu sondieren. Zweitens wurde die Ansicht bekräftigt, daß der Ausbau der Exportindustrie nach dem Krieg eine vordringliche Aufgabe der Wirtschaftspolitik sein müsse. Schließlich stellten die Minister auch eine Überprüfung der währungspolitischen Abkommen innerhalb des Sterlinggebiets in Aussicht ${ }^{38}$.

Keynes' Ausführungen und die sich daran anschließende Debatte im Kabinett wirkten vor allem im Foreign Office weiter. Einem Vorschlag Coulsons folgend, berief Sir Orme Sargent Anfang August 1944 eine Besprechung der Abteilungsleiter ein, um die Konsequenzen der in Keynes' Memorandum skizzierten Lage für die britische Außen-

\footnotetext{
34 CAB 65/43, W.M. 93 (44), War Cabinet Meeting, 18.7.1944.

35 Ebenda.

36 BLPES, Dalton Diaries I/30, fol. 177 verso (Eintrag 21.6.1944).

37 NC, MSS Cherwell, H 174, Anderson an Cherwell, 12.6.1944.

${ }^{38}$ CAB 65/43, W.M. 93 (44), War Cabinet Meeting, 18.7.1944.
} 
politik zu erörtern ${ }^{39}$. Sargent gab eine schonungslose Lageanalyse. Die finanzielle Situation Großbritanniens bei Kriegsende könne sehr wohl zu einem Kurswechsel der britischen Diplomatie zwingen: „We could no longer rely on the weapons of the rich man such as credits, loans and subsidies“, erklärte er, ”and we must get used to the position of a debtor nation. " Die Beamten äußerten zwar die Hoffnung, die USA werden als möglicher Kreditgeber einspringen, hielten dem jedoch sofort entgegen, daß damit ja eine Ausweitung des amerikanischen Einflusses einhergehe und möglicherweise gar der Grund für einen anglo-amerikanischen Konflikt heraufbeschworen werden könne. Als Ausweg aus diesem Dilemma erschien ihnen zu diesem Zeitpunkt nur die effiziente und gezielte Nutzung der spärlichen Ressourcen im Sinne der auch von Anderson geforderten Prioritätensetzung; Schatzamt und Handelsministerium sollten gleichzeitig angehalten werden, über mögliche Kompensationsmittel zur Erweiterung des außenpolitischen Handlungsspielraums nachzudenken. Im Foreign Office selbst war man sich einig, in Zusammenarbeit mit den einzelnen Ressorts ein Richtlinienpapier auszuarbeiten, das auch die möglichen Konsequenzen für die britische Deutschlandpolitik umfaßte. Bevor die Beamten sich aber noch an erste Entwürfe heranwagen konnten, veränderten weitere Entwicklungen die Außenbedingungen ihrer Planungsarbeit.

Im September 1944 traf sich Churchill, wie bereits geschildert, mit Präsident Roosevelt in Quebec zu Gesprächen. Die beiden Regierungschefs erörterten dabei nicht nur die Grundlagen ihrer Deutschlandpolitik (Morgenthauplan), sondern einigten sich auf den Rahmen der Lend-Lease-Lieferungen während der Phase zwischen der Kapitulation Deutschlands und dem Ende des Krieges mit Japan ("Stage II“). Die Details wurden erneut von Keynes ausgearbeitet, wobei ihm die Verhandlungsführung in geradezu frustrierender Weise von Lord Cherwell streitig gemacht wurde ${ }^{40}$. OCTAGON diente Churchill dazu, Roosevelt und den amerikanischen Stabschefs die Bereitschaft Großbritanniens zu erklären, in den Krieg mit Japan einzutreten, um so weitere Lend-LeaseMittel zu sichern, während London gleichzeitig nach dem Kriegsende in Zentraleuropa mit dem allmählichen Truppenabbau und dem Übergang auf eine Friedenswirtschaft beginnen sollte. Der Kriegspremier erklärte Roosevelt denn auch unumwunden, es sei wesentlich, daß die USA an Lend-Lease keine Bedingungen knüpften, die den Aufbau der britischen Exportindustrie bedrohten ${ }^{41}$. Zwar stimmte der Präsident in Quebec Churchill zum Entsetzen sowohl der amerikanischen Chiefs of Staff, die Verzögerungen bei der Lieferung kriegswichtiger Güter in den pazifischen Kriegsschauplatz befürchteten, als auch von Außenminister Hull, der die Zusage konditionsloser Hilfe in Höhe von \$6,5 Milliarden an Großbritannien scharf kritisierte, beidem prinzipiell zu, so daß die Fortschreibung von Lend-Lease über „VE-Day“ hinaus zunächst gesichert schien. Bei den sich unmittelbar an OCTAGON anschließenden Verhandlungen ließen die Amerikaner Keynes jedoch in keinem Zweifel, daß die Hilfe sich nur auf Kriegsmaterial beziehen und unter keinen Umständen den Charakter indirekter Wiederaufbauhilfe annehmen würde. Namentlich die amerikanische Industrie und ein wachsamer Kongreß, die die unlautere Nutzung der Materialien durch einen Handelskonkurrenten witterten,

\footnotetext{
${ }^{39}$ FO 371/40952/UE 615, Note of a Meeting held in Sir Orme Sargent's Room on 3rd August, 1944; dort auch das folgende Zitat.

${ }^{40}$ Vgl. Harrod, Keynes, S. 586-591; Moggridge, Maynard Keynes, S. 775 f., 778.

${ }^{41}$ Gilbert, Road to Victory, S. 954-970, besonders S. 964; Taylor, S. 711.
} 
drängten darauf, daß Lend-Lease streng am Bedarf des Krieges gegen Japan orientiert sein und auf diesen Zeitraum beschränkt werden sollte ${ }^{42}$. Die Niederwerfung der Achsenmächte war eines - Wiederaufbauhilfe ein anderes Kapitel. Und hatten die USA nicht gerade mit den Vereinbarungen von Bretton Woods ihren Beitrag zur wirtschaftlichen Rekonstruktion Europas geleistet? Die Hoffnung der Briten, die in der Folge der Bretton-Woods-Vereinbarungen um so dringlichere Sonderhilfe auf dem Wege von LendLease zu bekommen, erfüllte sich also nicht und lenkte die Aufmerksamkeit der Planer in London sofort auf „Stage III“. In einem die anstehenden ökonomischen Probleme treffend artikulierenden Sarkasmus nannte Keynes diese Phase "that happy period when we should be fighting neither Germans nor Japanese, and merely facing economic ruin" ${ }^{43}$.

In Quebec paraphierten Churchill und Roosevelt aber auch den Morgenthauplan. Wenn Keynes darum bemüht war, Klarheit gerade über den Zusammenhang zwischen ökonomischem Wiederaufbau und der Frage der wirtschaftlichen Behandlung Deutschlands nach dem Krieg zu gewinnen, so war dieses Vorhaben durch die Initiative des amerikanischen Finanzministers zunächst wirksam durchkreuzt, auch wenn der Plan nie eine echte Chance zur Verwirklichung hatte. Aus Keynes' Sicht mußte es jedenfalls wie ein Rückfall in die Zeit nach dem Ersten Weltkrieg erscheinen, daß ausgerechnet zu einem Zeitpunkt, als jenseits aller vordergründigen Erfolge von OCTAGON die britischamerikanischen Finanzgespräche die Probleme von "Stage III" mit besonderer Dringlichkeit aufwarfen, ein Konzept deutschlandpolitisch akut wurde, das dem erfolgreichen wirtschaftlichen Wiederaufbau Kontinentaleuropas und Großbritanniens prinzipiell diametral entgegengesetzt war. Wie schon zuvor die Ergebnisse der Konferenz von Bretton Woods bewertete Keynes auch OCTAGON und die Lend-Lease-Verhandlungen als Teilerfolg, dessen eigentlicher Wert darin lag, die Amerikaner zur Fortsetzung der Hilfe für Großbritannien verpflichtet zu haben; darüber hinaus tat man in London gut daran, die Öffentlichkeit von den positiven Aspekten der Gespräche zu überzeugen: der Umfang der Lieferungen sei zumindest nicht gekürzt worden, und auch die Freiheit, frühzeitig mit dem Ausbau der Exportindustrie zu beginnen, werde nicht eingeschränkt ${ }^{44}$. Gleichzeitig wußte er, daß die US-Hilfe zeitlich an den Krieg gegen Japan gebunden war und keineswegs die strukturellen Probleme des britischen Wiederaufbaus in der Übergangsphase einer Lösung näherbrachte. Sie konnte bestenfalls dazu dienen, das Land weiter auf der Basis des „muddle through" durch die Widrigkeiten der Nachkriegszeit zu steuern. Nahm man Bretton Woods und OCTAGON als Vorzeichen des Kommenden, so war damit kaum die so dringend notwendige und von Keynes mühsam vorskizzierte deutschlandpolitische und weltwirtschaftliche Weichenstellung vorgenommen worden. Seine ganze Kassandra-Natur offenbarend, notierte Keynes: „We cannot police half the world at our own expense when we have already gone into pawn to the other half. We cannot run for long a great programme of social amelioration on money lent from overseas. Unless we are willing to put ourselves financially at the mercy of America and then borrow from her on her own terms and conditions sums which we cannot confidently hope to repay, what are we expecting? Are we looking forward to a

\footnotetext{
42 Vgl. Gardner, Sterling-Dollar Diplomacy, S. 180-182; Gaddis, S. 120.

${ }^{43}$ Harrod, Keynes, S. 587.

${ }^{44}$ BLPES, Dalton Diaries I/31, fol. 97 verso (Eintrag 14.12.1944).
} 
spectacular bankruptcy (not, altogether, a bad idea) from which we shall rise next morning without a care in the world? Or are we following some star at present invisible to me?"45

Diese Bemerkungen sind einer Kabinettsvorlage entnommen, die Keynes im März 1945, etwa einen Monat nach der Konferenz von Jalta, erarbeitete und in der er den Bedingungen der britischen Wirtschaftspolitik in "Stage III“ nachging. Diese Denkschrift lag dem Kabinett Mitte Mai vor. Als Alternative zu einer sowohl für Großbritannien als auch für den europäischen Wiederaufbau gefährlichen ökonomischen Isolation des Landes sah Keynes nur noch die Möglichkeit, mit Washington über die Bedingungen einer umfangreichen Finanzhilfe zu verhandeln. Er griff seinen zunächst in dem Memorandum vom Juni des Vorjahres ausgebreiteten Gedanken auf, daß nur die USA die Devisenlücke Großbritanniens füllen könnten. Erstmals ventilierte er nun den Gedanken, Washington die Gewährung eines nic htrückzahlbaren Darlehens vorzuschlagen und ihm im Gegenzug diese bittere Pille durch die Ankündigung zu versüßen, den Sterling binnen Jahresfrist nach Beendigung des Krieges frei konvertibel zu machen. Wie er ausführlich und in drastischen Worten darlegte, war dieses Quid pro quo-Geschäft das ÄuBerste, was unter den gegebenen Umständen erwartet werden durfte, und obwohl unter den Beamten des Schatzamts wie auch in der Führungsspitze durchaus Widerspruch anzutreffen war, setzte sich im Laufe des Sommers in Whitehall der Gedanke als britische Maximalposition für „Stage III“ durch.

\section{„Policy of Selection": Eine neue außenpolitische Strategie}

Auch im Außenministerium hatten sich die durch Keynes' Anregungen ins Rollen gebrachten Arbeiten für ein Grundsatzpapier über den Zusammenhang von Wirtschaft und Diplomatie mittlerweile konkretisiert. Zwischen September 1944 und Januar 1945 entstanden eine Reihe von Vorfassungen ${ }^{46}$, die von der Wirtschaftsabteilung des Foreign Office und dem Schatzamt in einer gemeinsamen Aktion bis Anfang Februar zu einer Vorlage zusammengefaßt werden konnten. Die Planungen im Rahmen der Konferenz von Jalta verzögerten jedoch weitere Diskussionen, so daß Außenminister Eden erst am 22. März mit der Prüfung begann. Das Memorandum erhielt nach einigen Änderungen Edens Zustimmung und wurde eine Woche später mit der Anweisung, es als "geheim“ zu behandeln und nur einem besonderen Personenkreis Einsicht zu gewähren, als Runderlaß an die diplomatischen Vertretungen weitergeleitet ${ }^{47}$.

Die Beamten ließen keinen Zweifel daran, daß ihre bitteren Erfahrungen der Zwischenkriegszeit, als die wirtschaftliche Krisenlage des Landes das Ressourcenfundament der britischen Diplomatie, insbesondere in Form eines adäquaten Rüstungsniveaus, ver-

\footnotetext{
45 Memorandum Keynes, "Decisions of Policy Affecting the Financial Position in Stages II and III“, undatiert, in: JMK XXIV, S. 114-126; hier: S. 125.

$46 \mathrm{Vgl}$. FO 371/40952/UE 615.

${ }^{47}$ FO 371/45694/UE 813, Minute J. E. Coulson, 9.2.1945; Sargent an Eden, 22.3.1945; Minute Eden, 25.3.1945; Entwurf des Begleitschreibens Edens, 30.3.1945.
} 
eitelt hatte, die eigentliche Motivationskraft hinter ihren Ausführungen bildeten ${ }^{48}$. Mit Blick auf die Nachkriegszeit wurde die Hoffnung formuliert, daß Großbritannien eine ausreichende militärische Stärke würde beibehalten können; die Beamten bekräftigten jedoch den Grundsatz, „that our diplomatic policy should be commensurate with our economic power. If we enter into commitments which our economic situation will not bear, we shall be exposed to another series of humiliations." Die voraussichtliche Devisenknappheit und das bedrohliche Zahlungsbilanzdefizit, notierten sie nüchtern, „will deprive our foreign policy of many weapons on which we relied in the past and which we would like to employ in the future. We shall find it difficult to grant credits, even where we might expect them to produce useful economic results in the long run. We shall definitely not be able to afford uneconomic purchases for political reasons. We shall have to go slow over subsidies, and we shall have to put a stop to many forms of investment in foreign countries. Every subscription to international organisations and charities will require the coldest scrutiny." ${ }^{49}$ Hier war der zu erwartende Einflußverlust zugunsten der USA klar formuliert. In dem Maße, wie sich Drittländer insbesondere ökonomisch an Washington anlehnten, ging Unterstützung für die Anliegen Großbritanniens verloren; dies betraf die machtpolitische Position des Königreichs genauso wie seine Einflußmöglichkeiten auf die weltwirtschaftliche Neuordnung. Gleichzeitig mußte die finanzielle Abhängigkeit Londons von Washington zunehmen, der Spielraum, innerhalb dessen die Amerikaner, wie Shackle vom Board of Trade es vier Jahre zuvor formuliert hatte, zum richtigen Ausspielen der Karten gebracht werden konnten, wenn man ihnen schon das bessere Blatt überlassen mußte, immer kleiner werden. Die strukturelle Abhängigkeit, die sich hinter der äußeren finanziellen verbarg, mußte besonders zu denken geben. Denn würden sich umgekehrt die USA einer wirtschaftlichen Zusammenarbeit verschließen - und damit theoretisch Großbritannien eine Führungsposition überlassen -, wäre es nicht nur um ein Vielfaches schwieriger, den notwendigen Ausbau des Außenhandels voranzutreiben und so rasch wie möglich zu normalen Verhältnissen zurückzukehren; vielmehr vergrößerten sich dann auch die Lasten, die Großbritannien als Führungsmacht im Rahmen der sicherheitspolitischen Konsolidierung Europas und des Commonwealth zu tragen haben würde. An Positiva war diesem Befund eigentlich nicht viel mehr entgegenzusetzen als der Prestigegewinn, der sich aus der Niederschlagung Hitlers ergab, und die traditionellen Handelskanäle zu den überseeischen Erzeugerländern.

Welche Konsequenzen hatte das aus der Perspektive des Foreign Office für die britische Außenpolitik der Nachkriegszeit? Unter der Voraussetzung, daß sich die Diploma-

\footnotetext{
${ }^{48}$ In einer aufschlußreichen, von Eden allerdings gestrichenen Passage, in der die Verfasser die ganze Hilflosigkeit der britischen Diplomatic rekapitulierten, hieß es: „The results were disastrous because our bluff was called. We could do nothing effective to check Italian aggression in Abyssinia. We had to connive at the flagrant intervention of Germany and Italy in the Spanish civil war. We allowed Germany to violate the clauses of the Versailles Treaty one after another and to annex Czechoslovakia. In a final attempt to recover our self-respect, we guaranteed the frontiers of Poland. Once again our bluff was called." FO 371/45694/UE 813, Memorandum J. E. Coulson, „The Effect of Our External Financial Position on our Foreign Policy“, 30.3.1945; dort auch die Eden vorgelegte Fassung mit dessen handschriftlichen Änderungen. Zu dem Memorandum vgl. auch Meyers, Economic Security, S. $55 \mathrm{f}$.

${ }^{49}$ FO 371/45694/UE 813, Memorandum J. E. Coulson, „The Effect of Our External Financial Position on our Foreign Policy", 30.3.1945.
} 
tie nicht primär am Wünschenswerten, sondern am Möglichen orientieren sollte bzw. umgekehrt bei möglichst optimaler Nutzanwendung der knappen Ressourcen so viel wie möglich von den außenpolitischen Zielvorgaben erreicht werden sollte, empfahlen die Beamten eine „policy of selection“. Darunter verstanden sie die gezielte Einsetzung der vorhandenen ökonomischen Mittel in ausgesuchten Gebieten des britischen Interessenbereichs zu dem Zweck der direkten Einflußnahme und sicherheitspolitischen Stabilisierung. Im Zentrum dieser „eingeschränkten Finanzdiplomatie" standen nach ihrer Ansicht China im Fernen Osten, Österreich und Griechenland in Südosteuropa ${ }^{50}$ sowie die Ölländer Saudiarabien und Persien ${ }^{51}$, deren finanzielle Unterstützung man in je unterschiedlicher Höhe für notwendig erachtete. Mit Bedauern stellten sie allerdings fest, daß ein Engagement auf dem Balkan und in Lateinamerika zwar wünschenswert sei, wohl aber um einiges die Finanzmittel des Königreichs übersteige. In bemerkenswerter Verkennung der tatsächlichen britischen Handelsstruktur und der späteren weltwirtschaftlichen Expansion hielten sie die bestehenden Handelsverbindungen zu den westeuropäischen Staaten und Skandinavien für ausreichend; hier versprachen sie sich von einer wirtschaftlichen Betätigung keinen ausreichenden außenpolitischen Einflußgewinn $^{52}$. Nach Ansicht der Planer von Foreign Office und Treasury sollte diese „policy of selection" flankiert werden von der allgemeinen Ausrichtung der britischen Außenpolitik nach dem Krieg an dem Ziel der Wiederherstellung ökonomischer Stärke: „The recovery of our economic strength depends first and foremost on the revival and expansion of our export trade. This point must always be in the forefront of our minds. Our industrialists, merchants and bankers should receive constant and active encouragement to foster our exports in every way open to them, and every proper facility which their representatives abroad may require should be given to them ungrudgingly and with promptitude. [...] As we are deprived of some of our former weapons, we must make more use of those we still possess. There must be the greatest possible development of friendly relations with economic circles in foreign countries. The despatch of economic business by the Foreign Service must be efficient, and its members must be capable at all times of giving good advice to foreign Governments on economic matters. It will be es-

${ }^{50}$ Zur Bedeutung Griechenlands vgl, auch Clark Kerr an Eden, 10.7.1945, in: DBPO I/1, S. 142148, hier: S. 146; Caccia an Churchill, 10.7. 1945, in: DBPO I/1, S. $154 \mathrm{f}$.

51 Zur Bedeutung Persiens vgl. Bullard an Eden, 11.7.1945, in: DBPO I/1, S. 168f.; Bullard an Eden, 11.7.1945, in: DBPO I/1, S. 169; Gainer an Perowne, 13.7.1945, in: DBPO I/1, S. 263-265; Eden an Churchill, 13.7.1945, in: DBPO I/1, S. $265 \mathrm{f}$.

$52 \mathrm{Im}$ Gegenteil warnte Coulson in einer früheren Version des Memorandums vor einem zu starken Engagement in Europa und verwies auf den amerikanischen Kapitalfluß nach Deutschland nach dem Ersten Weltkrieg, der nach allgemeiner Meinung der Außenamtsbeamten das Negativbeispiel par excellence für eine verfehlte Außenwirtschaftspolitik darstellte. Coulson empfahl, ein Auge auf eine mögliche Neuauflage amerikanischer Kapitalinvestitionen in Deutschland nach dem Krieg zu werfen. „We are, of course, committed by our public pronouncements to enabling the Germans to work and to enabling Germany to have an economic, if not a military future [sic!]." Hinsichtlich einer Stabilisierungspolitik gegenüber Deutschland erhob er Einspruch "against the repetition of the policy carried out by the City after the last war, with such disastrous effects, of pouring money into Germany in order to set her on her feet again. If there is to be any repetition of this policy I take it that it will come from Wall Street and I hope we shall exert all our influence to ensure the observance of the principle that the needs of our allies come first, those of the neutrals second, and those of Germany and our enemies last." Vgl. den Entwurf in FO 371/ 40952/UE 615. 
sential that our diplomatic missions pay the greatest attention to all these aspects of their work." 53

Reaktionen auf das Grundsatzpapier ließen nicht lange auf sich warten. Umgehend wies ein frankophiler Beobachter der internationalen Entwicklung wie der britische Botschafter in Paris, Duff Cooper, auf zwei aus seiner Sicht gravierende Schwachstellen des Memorandums hin ${ }^{54}$. Ende Juni 1945 hatte ihn der französische Außenminister Georges Bidault erneut auf die Dringlichkeit eines britisch-französischen Bündnisvertrages hingewiesen, so daß der Inhalt der Denkschrift sich mit der laufenden Diskussion über die bereits von Spaak lancierte Idee eines regionalen Sicherheitspaktes verband ${ }^{55}$. Cooper wies die Zentrale in London darauf hin, daß erstens der Stellenwert Kontinentaleuropas für Wirtschaft und Sicherheit Großbritanniens sträflich unterbelichtet werde und es zweitens kaum ausreichen könne, wenn man in London lediglich über die Möglichkeiten einer eingeschränkten Finanzdiplomatie gegenüber den britischen Interessengebieten nachdenke. Hier, so erschien es Cooper, waren keineswegs alle notwendigen Konsequenzen aus Großbritanniens wirtschaftlicher Situation gezogen. Er stellte in Frage, ob England unter den gegebenen Umständen sich weiterhin auf seine EmpireRolle konzentrieren könne. Gerade die durch den Krieg erzwungene ökonomische Entflechtung und die damit einhergehende machtpolitische Depotenzierung in Übersee warf für ihn die Frage auf, ob ein stärkeres Engagement in Europa nicht nur politisch wünschenswert, sondern auch notwendig sei. War es denn nicht geradezu unerläßlich, daß England auch in Europa eine wirtschaftlich stärkere Führungsrolle akzeptierte? Und mußte diese Führungsrolle nicht auch auf die militärischen und politisch-kulturellen Bereiche erweitert werden? In Frankreich, berichtete der um ein vertragliches Zusammengehen mit Frankreich bemühte Botschafter Cooper ${ }^{56}$, werde offen darüber nachgedacht, in welcher Richtung man ein als notwendig erachtetes wirtschaftliches und sicherheitspolitisches Zusammenrücken der europäischen Nachbarländer vorantreiben solle. In diesem Zusammenhang würden Vorschläge zur Schaffung einer Zollunion laut ${ }^{57}$. Namentlich in Geschäftskreisen werde ein Zusammenschluß der europäischen Industrien favorisiert. Natürlich, so fügte Cooper hinzu, bedeute dies nichts anderes als den Aufbau eines „Trusts" und führe so zu jener Kartellisierung, vor der sich Engländer und Amerikaner so sehr fürchteten und deren Entstehung in der Nachkriegszeit die USA unter allen Umständen verhindern wollten ${ }^{58}$; Industrielle in Frankreich wüßten jedoch, daß das Land seine Geschäftsgrundlagen auf eine breitere Basis stellen müsse. "What seems certain", stellte er fest, „is that, faced as they [the French] are with a constant upward pressure on wages, the prospect of having to devalue their currency and other problems affecting social and labour policy at home and in their empire, the French, nervous of their eastern neighbours and distrustful of the United States, look to the possibility of an arrangement with the Western European Powers and with the Unit-

${ }^{53}$ FO 371/45694/UE 813, Memorandum Coulson, „The Effect of Our External Financial Position on Our Foreign Policy“, 30.3.1945.

${ }_{54}$ FO 371/45694/UE 2504, Cooper an Eden, 12.6.1945.

55 Brief for United Kingdom Delegation at Terminal [Potsdamer Konferenz]: „The ,Western

Group "and Franco-British Treaty“, 10.7.1945, in: DBPO I/1, S. 235-241, hier: S. 239.

${ }^{56} \mathrm{Vgl}$. Young, S. $45 \mathrm{f}$; Ebersold, S. 101, $136 \mathrm{f}$.

57 Vgl. oben Kap. V.2.

${ }^{58} \mathrm{Vgl}$. Berghahn, Unternehmer und Politik, S. 20-39 passim, 84 ff., 115-117. 
ed Kingdom which could provide the broad basis which is indispensable for their economy. "59 Um den Verdacht der ökonomischen Blockbildung auszuräumen, aber auch um Großbritannien mit seiner Sonderstellung gegenüber den USA und innerhalb des Empire die Übernahme einer Führungsrolle zu erleichtern, ergänzte Cooper, könnte ein solches regionales Abkommen in den Zusammenhang eines übergeordneten Paktsystems gestellt werden. Regionalsysteme einerseits und offener Weltmarkt andererseits, so sein Plädoyer, stünden sich nicht als unvereinbare Gegensätze gegenüber. Gerade der ordnungspolitische Wert regionaler Zusammenschlüsse für einen raschen ökonomischen Wiederaufbau mußte seiner Meinung nach auch skeptische Amerikaner davon überzeugen, daß der Zusammenschluß der europäischen Wirtschaft als Subsystem ein wichtiger Motor für die Regeneration des Welthandels auf multilateraler Basis sein konnte. Für Cooper war diese Entwicklung unabwendbar, und er wies London deshalb darauf hin, daß eine "policy of selection" weder an Kontinentaleuropa vorbeigehen noch sich auf mögliche finanzielle Hilfestellungen beschränken dürfe. Im Gegenteil warnte er seine Vorgesetzten, daß der Zusammenschluß der westeuropäischen Industrien auch ohne britische Beteiligung vorangetrieben werden würde. Für diesen Fall schloß er nicht aus, daß die ökonomische Integrationsbewegung sich in einer für die Interessen Londons nachteiligen Weise entwickeln und etwa ein wirtschaftliches Rivalitätsverhältnis zur britischen Industrie begründen konnte. Sollte ein solches Bündnis gar unter den Einfluß der Sowjetunion kommen, würde sich die Inselmacht erneut vor einer geradezu lebensbedrohenden Herausforderung sehen: „Unless the basic problem is tackled, namely, the revival of industry and distribution and the placing of various countries on a sound economic and financial basis, there can be no stability in Europe, and without this stability the future for the world is hopeless. The United Kingdom is the only country in the West with sufficient prestige to give a lead which will prevent a relapse into economic and political nationalism, or alternatively [...] to threatening combinations. "60

Coopers Ausführungen, unterstützt durch Pressekommentare, die auch im Außenministerium Widerhall fanden ${ }^{61}$, gaben den Anstoß für eine interministerielle Besprechung am 25. Juli 1945, also genau einen Tag vor Bekanntgabe der Ergebnisse der Parlamentswahlen, die der Labour Party eine absolute Mehrheit brachten. Der Zeitpunkt war keinesfalls günstig, denn trotz des erfolgreichen Eintretens Churchills in Jalta für eine französische Beteiligung an der Besetzung Deutschlands und Sondierungsversuchen von Außenminister Bidault bei Cooper zeigten sich die britisch-französischen Beziehungen von Problemen überschattet. Die Ursache hierfür dürfte wohl hauptsächlich bei de Gaulle gelegen haben. Dessen in den Augen Londons überhebliches Großmachtdenken und seine anglophobe Einstellung fand die britische Entscheidungsspitze erneut bestätigt, als er im Februar 1945 seinen Außenminister in die britische Hauptstadt schickte, um die Bedingungen für ein englisch-französisches Bündnis vorzutragen: Einigung über Syrien und den Libanon im französischen Sinn; gleiche Beteiligung Frankreichs an der Besetzung Deutschlands, insbesondere eine in der Größe der englischen und amerikani-

59 FO 371/45694/UE 2504, Cooper an Eden, 12.6.1945.

60 Ebenda.

${ }^{61}$ Vgl. z. B. die beiden Artikel „Britain in Europe“ von Walter Hill in der Financial News vom 28. und 29.6.1945. 
schen vergleichbare Besatzungszone; sowie die Versicherung Londons, an keiner Konferenz mit Russen und Amerikanern teilzunehmen, zu der nicht auch Paris eingeladen werde ${ }^{62}$. Diese Offerte stieß an der Themse auf Unverständnis, ja Empörung und trug wesentlich dazu bei, die britisch-französische Annäherung in der Bündnisfrage auf Eis zu legen ${ }^{63}$. Schließlich entsprach Coopers ökonomische Argumentationsweise auch kaum dem Foreign Office-Konsens, daß sich ein Regionalzusammenschluß nicht gegen die Sowjetunion wenden dürfe. Wirtschaftliche Gründe für ein Engagement Großbritanniens auf dem Kontinent mochten aus der Sicht Whitehalls für eine interessante zusätzliche Note sorgen, wurden jedoch eindeutig vom Primat des außenpolitischen Denkens überdeckt, der in diesem Zusammenhang von Eden gegenüber Spaak bereits ausführlich erläutert worden war.

Mit dieser Ansicht vermochten sich die Außenamtsbeamten schließlich auch gegenüber den Kollegen aus den Wirtschaftsressorts in der Frage eines westeuropäischen Regionalpakts unter britischer Beteiligung durchzusetzen. In der Besprechung des Außenministeriums am 25. Juli 1945 mit Vertretern des Schatzamts, des Handelsministeriums, des Department of Overseas Trade, der Cabinet Offices und der britischen Botschaft in Paris war man sich schließlich einig, daß ein derartiger Zusammenschluß und als Voraussetzung hierfür ein britisch-französisches Bündnis zwar langfristig wünschenswert sei$\mathrm{en}^{64}$. Als Gründe machte man vornehmlich die politischen und strategischen Vorteile geltend, die ein solcher Zusammenschluß aus der Sicht Großbritanniens zu bringen versprach. Ökonomische Vorteile zog man nur insoweit ins Kalkül, als ein Sicherheitsbündnis eine gewisse Standardisierung der Rüstung und infolgedessen eine internationale Arbeitsteilung bzw. Rationalisierung der nationalen Rüstungsindustrien mit sich bringen würde. Dies markierte offensichtlich für London auch die Grenze der Mitarbeit bei einem wirtschaftlichen Zusammenschluß Westeuropas. Darüber hinaus konnte man sich nur allgemein darauf verständigen, Maßnahmen zu unterstützen, die dem Aufbau eines multilateralen Handelssystems dienten, um so einer kontinentaleuropäischen Kartellisierung vorzubeugen; die Mitarbeit in einer Zollunion europäischer Provenienz schlossen die Teilnehmer der Besprechung aus. Der Entwurf eines Vertrages zwischen Großbritannien und Frankreich, der den Instruktionen für die britische Delegation bei der Potsdamer Konferenz für den Fall beigefügt wurde, daß die Frage eines westeuropäischen Bündnisses aufkam, konzentrierte sich dementsprechend auf die Verpflichtung zu gegenseitigem Beistand für den Fall erneuter deutscher Aggression; gleichzeitig war der Ausbau der Allianz zu einem regionalen Sicherheitssystem mit Frontstellung gegen Deutschland vorgesehen. Bezüglich der wirtschaftlichen Zusammenarbeit hielt der Ent-

62 Rothwell, S. 410; Young, S. 43. Zu den Meinungsverschiedenheiten zwischen London und Paris über die Levante vgl. Holman an Eden, 7.7.1945, in: DBPO V1, S. 30 f.; Brief for the United Kingdom Delegation to the Conference at Potsdam, in: DBPO I/1, S. 115-117; Eden an Halifax, 10.7.1945, in: DBPO I/1, S. 119f., Henderson an Francfort, 12.7.1945, in: DBPO I/1, S. 204; Cooper an Eden, 13.7.1945, in: DBPO I/1, S. 252; Anderson an Shone, 15.7.1945, in: DBPO I/1, S. 292-294; Eden an Lord Killearn, 16.7.1945, in: DBPO I/1, S. 336.

${ }^{63} \mathrm{Vgl}$. Brief for the United Kingdom Delegation to the Conference at Potsdam, 12.7.1945, in: DBPO I/1, S. 234-251, hier: S. 239.

${ }^{64}$ FO 371/45694/UE 3621, „Economic Relations with France and Western Europe“. Minutes of a Meeting held at the Foreign Office on 25th July, 1945. 
wurf lediglich die Willensbekundung fest, sich nach der Niederschlagung Deutschlands gegenseitig wirtschaftliche Hilfe zu leisten ${ }^{65}$.

Die Frage eines britisch-französischen Beistandspaktes, die seit 1941 in den Kriegszieldiskussionen kontinuierlich schwelte und über die nun im Zusammenhang mit den deutschlandpolitischen Entscheidungen von Jalta und Potsdam sowie dem Grundsatzpapier des Foreign Office über die Auswirkungen der wirtschaftlichen Situation Großbritanniens auf die Außenpolitik eine Vorentscheidung gefallen war, bestätigte erneut eindrucksvoll den Grundkonsens, der sich in den Reihen des Außenministeriums gegen Ende des Krieges herausgebildet hatte: Wie schon in der Reparationsfrage nahm man die wirtschaftliche Situationsbeschreibung der Experten unter Keynes' Leitung mit einiger Sorge zur Kenntnis und formulierte auch im Bereich der Sicherheits- und Bündnispolitik dem „First charge“-Prinzip analoge Grundsätze, die den Traditionspfad der Außenpolitik aber nicht verließen, sondern lediglich Anpassungen im Detail zuließen und sich an den tagespolitischen Erfordernissen orientierten; eine strukturelle Neuorientierung der Außenpolitik fand jedoch auf diesem Wege nicht statt. In diesem Sinne waren die programmatischen Äußerungen Coulsons und seiner Beamtenkollegen aus den anderen Ministerien, die den wachsenden Stellenwert ökonomischer Faktoren für die britische Außenpolitik in der Nachkriegszeit ausdrücklich anerkannten, in ihrer Bedeutung kaum zu unterschätzen. Insofern sie allerdings aus der voraussichtlichen wirtschaftlichen $\mathrm{Si}$ tuation des Landes bei Kriegsende im wesentlichen den Schluß zogen, daß der Spielraum für eine Finanzdiplomatie Londons im Sinne einer wirtschaftlichen Einflußnahme auf die Entwicklung in der britischen Interessensphäre eingeschränkt war, behielten sie den Blickwinkel des Primats der Außenpolitik bei und modifizierten vereinzelt traditionelle Mittel der Außenpolitik mit Bezug auf gerade Aktualität beanspruchende Fragen der äußeren Entwicklung. Entscheidungsleitend war die Frage, wie das an einem Primat der Außenpolitik orientierte diplomatische Instrumentarium unter den gegebenen Bedingungen angepaßt werden konnte, und nicht, ob der ökonomische Wandel auch ein Umdenken hinsichtlich der Grundlagen der britischen Außenpolitik generell erforderte.

\section{Ein finanzielles Dünkirchen? Keynes' Lageanalyse vom August 1945}

Mit dem Ende der Potsdamer Konferenz am 2. August $1945^{66}$ war auch die dringende Frage nach der Finanzierung des britischen Wiederaufbaus in der Übergangszeit erneut aufgeworfen. Präsident Truman hatte in einem Schreiben Churchill darauf hingewiesen, daß die britischen Gold- und Devisenvorräte sich in einem Maße verbessert hätten, daß London durchaus in der Lage sei, einen größeren Anteil an den noch zu leistenden Kriegskosten selbst zu tragen; Washington bekundete damit offen seine Sorge, daß man an der Themse die Lend-Lease-Mittel weniger zur Kriegsfinanzierung als zur Sanierung der Devisenkasse auf amerikanische Kosten ausgab. Bereits Anfang Juli 1945 hatten die britischen Stabschefs das Kabinett dahingehend alarmiert, „that the machinery of assignment [of Lend Lease] is virtually at a standstill“"67. Am Rande der Konferenz war es deshalb auch zu Vorverhandlungen über die Fortführung des Hilfsabkommens im Krieg

${ }^{65}$ Draft Treaty of Alliance and Mutual Assistance between the United Kingdom and the French Republic, in: DBPO I/1, S. $248 \mathrm{f}$.

${ }^{66}$ Zur Potsdamer Konferenz vgl, unten Kap. VII.1.

${ }^{67}$ Joint Staff Mission (Washington) an Cabinet Office, 7.7.1945, in: DBPO I/1, S. 39 f., hier: S. 40. 
mit Japan zwischen den amerikanischen und britischen Stabschefs sowie den Diplomaten beider Delegationen gekommen, die allerdings eher die Divergenzen beider Auffassungen offenbarten ${ }^{68}$. Gleichzeitig erhöhten die Potsdamer Entscheidungen über die Maßnahmen zur wirtschaftlichen Kontrolle Deutschlands, die auch in London nicht kritiklos aufgenommen wurden, sowie die gerade in der Deutschlandfrage deutlich zutage tretenden Meinungsverschiedenheiten mit Moskau den Druck, möglichst rasch mit Washington zu einer Einigung über die Fortschreibung von Lend-Lease und die Gewährung von Sonderkrediten für die Aufbauphase nach Beendigung des Krieges mit Japan („Stage III“) in dem von Keynes im März dieses Jahres dargelegten Sinn zu kommen.

In Potsdam hatte Truman Premierminister Attlee schließlich wissen lassen, daß er sofort nach Beendigung der Konferenz Assistant Secretary of State William Clayton nach London zu einer Sitzung der United Nations Relief and Rehabilitation Administration (UNRRA) entsenden werde; bei dieser Gelegenheit erwarte er ebenfalls einen ersten britisch-amerikanischen Meinungsaustausch über Lend-Lease nach Beendigung des Krieges gegen Japan ${ }^{69}$. Bei seinem Zwischenstop bekräftigte Clayton gegenüber Keynes den amerikanischen Standpunkt, daß eine Einigung in den Finanzgesprächen untrennbar mit einem Abkommen über die handelspolitischen Grundlagen der Nachkriegszeit verbunden sein werde; mit anderen Worten, amerikanische Finanzhilfe würde sowohl von weiteren währungs- als auch von handelspolitischen Zugeständnissen Londons abhängen ${ }^{70}$. Im Verlaufe eines weiteren Gesprächs mit Vertretern von Schatzamt, Handelsministerium und der Economic Section erklärte Clayton, daß Großbritannien zugleich nicht zu viele Hoffnungen auf allgemeine Zollreduzierungen der USA hegen dürfe; er hielt selbst eine geringfügige Lockerung der Importbestimmungen um $20 \%$ für ausgeschlossen. Auch biete der erst kürzlich erneuerte Reciprocal Trade Agreements Act ${ }^{71}$ dem Präsi-

${ }^{68}$ Rowan an Churchill und Memorandum Truman, 17.7.1945, in: DBPO L/1, S. 350f.; Surmmarized Note of the Prime Minister's Conversation with President Truman at Luncheon on 18 July 1945 , 18.7.1945, in: DBPO I/1, S. 367-371, besonders S. $368 \mathrm{f}$.; Report by the British Chiefs of Staff to Mr. Churchill, 20.7.1945, in: DBPO I/1, S. 438-442; Memorandum by the British Chiefs of Staff to Mr. Churchill, in: DBPO I/1, S. $558 \mathrm{f}$.; Memorandum by the British Chiefs of Staff, „LendLease", 23.7.1945, in: DBPO I/1, S. 559 f.; Minutes of a British Chiefs of Staff Conference, 23.7.1945, in: DBPO 1/1, S. 572f.; Churchill an Truman, 24.7.1945, in: DBPO I/1, S. 613 f.; Anderson an Eden, 18.7.1945, in: DBPO I/1, S. 749 f.; Sargent an Cadogan, 20.7.1945, in: DBPO I /1, S. 792 f.; Truman an Attlee, 29.7.1945, in: DBPO 1/1, S. 988 f.; Attlee an Truman, 31.7.1945, in: DBPO I/1, S. 1097; BL Add Mss 52578, Cunningham Papers, Diaries, 23.7.1945.

${ }^{69}$ Truman an Attlee, 29.7.1945, in: DBPO I/1, S. $988 \mathrm{f}$.

70 Vgl. Harrod, Keynes, S. 593.

${ }^{71}$ Am 5. Juli 1945 hatte der amerikanische Kongreß eine Modifizierung des Handelsgesetzes aus dem Jahre 1934 beschlossen, das seinerseits auf den Bestimmungen von 1930 aufbaute. Der Präsident wurde autorisiert, beim Abschluß bilateraler Handelsverträge ausgewählte Zölle bis maximal 50\% zu senken oder anzuheben. Diese vor allem die amerikanische Handelspolitik der dreißiger Jahre bestimmende Praxis hatte zum Ziel, auf der Basis von Bilateralität die Präferenzsysteme im Welthandel aufzuweichen und auf das Prinzip der Reziprozität zu verpflichten. Vgl. den Überblick bei Smith, Art. „Reciprocity“, S. 878b-879a. Zur Bedeutung des Reciprocal Trade Agreements Act im Kontext der US-Außenpolitik, insbesondere der deutsch-amerikanischen Beziehungen, vgl. Schröder, Deutschland und die Vereinigten Staaten, S. 135-143. Am gleichen 
denten keine Möglichkeiten, die zollpolitische Prärogative des Kongresses zu umgehen und eine umfassende Verminderung der Zollbarrieren einzuleiten. Als weitaus günstigere Instrumente betrachte man in Washington den Abschluß einer Reihe bilateraler Handelsabkommen, die im Rahmen einer umfassenden Handelskonferenz von den Teilnehmerländern untereinander abgeschlossen werden könnten ${ }^{72}$.

Der Druck auf London nach einer Lösung der anstehenden finanztechnischen Probleme sowie nach wirtschaftspolitischen Grundsatzentscheidungen für die unmittelbare Nachkriegsphase wuchs weiter, als in derselben Woche die japanische Regierung ihr Kapitulationsangebot übergab und zugleich im Außenministerium erste Hinweise auf eine mögliche Einstellung von Lend-Lease durch die Amerikaner eintrafen ${ }^{73}$. Am 14. August 1945 sandte der neue Schatzkanzler Hugh Dalton schließlich ein weiteres ausführliches Memorandum von Keynes an die Mitglieder des Kabinetts, das die Diskussionen der letzten Monate im Schatzamt zusammenfaßte und pointiert und in gewohnter Schärfe ein äußerst düsteres Zukunftsbild für Großbritannien zeichnete ${ }^{74}$. Die einfache Gleichung, die Keynes zunächst aufmachte, war atemberaubend: Wenn alle Formen der Unterstützung - also Lend-Lease aus den USA, Devisen aus dem Sterling-Gebiet und die Hilfe der kanadischen Regierung - bis zum Jahresende weiterliefen, so fülle dies ein Defizit in Höhe von $£ 2,1$ Milliarden, für das nach Auslaufen der kriegsgebundenen Hilfen keine Deckung mehr bestünde. Das britische Exportvolumen war auf ein Drittel des Vorkriegsstandes gefallen, seinen Wert schätzte Keynes für das laufende Jahr auf nicht mehr als $£ 350$ Millionen 75 ; die Einkünfte aus den „unsichtbaren“ Exporten beliefen sich seiner Meinung nach lediglich auf rund $£ 100$ Millionen $^{76}$. Wichtige Devisenquellen wie etwa die Ausgaben der US-Soldaten auf britischem Boden und die Zahlungen der Dominions für den Unterhalt ihrer Truppen (zusammen auf $£ 350$ Millionen veranschlagt), fielen ebenfalls weg. Die Gold- und Devisenvorräte waren auf $£ 500$ Millionen geschrumpft. Die Schulden hatten sich im Verlaufe des Krieges nach Keynes' Schätzungen auf $£ 3$ bis 4 Milliarden hochgeschraubt, "which we shall be owing to almost every country in the world" "77. Auf der Ausgabenseite wuchs der Importbedarf an Nahrungsmitteln und Rohstoffen, um insbesondere das im Zuge der Demobilisierung sich schwerer ge-

Tag hatte Truman in einer Direktive für das Kriegs- und Marineministerium verfügt, daß alle weiteren Lend-Lease-Lieferungen künftig auf die ausschließlich für den Krieg gegen Japan notwendigen Materialien zu beschränken seien. Vgl. Joint Staff Mission an Cabinet Office, 7.7.1945, in: DBPO I/1, S. 39 f.; Report by the British Chiefs of Staff to Mr. Churchill, 20.7.1945, in: DBPO I/1, S. 438-440.

72 Summary of Discussion held at the Board of Trade on 4 August 1945, in: DBPO I/3, S. 6-19. Vgl. zum Konzept einer „multilateral-bilateralen“ Handelspolitik Gardner, Sterling-Dollar Diplomacy, S. 150-153.

${ }_{73}$ Vgl. z. B. Balfour an Bevin, 11.8.1945, in: DBPO I/3, S. 23-25; Balfour an Bevin, 14.8.1945, in: DBPO I/1, S. $26 \mathrm{f}$.

${ }^{74} \mathrm{CAB} 129 / 1, \mathrm{CP}$ (45) 112, Memorandum Keynes, „Our Overseas Financial Prospects“, 14.8.1945; DBPO I/3, S. 27-37; JMK XXIV, S. 398-411.

75 Tatsächlich erreichten die Exporte, inclusive Re-exporte, die Höhe von $£ 450$ Millionen. Vgl. Feinstein, Statistical Tables, T 82 (Tab. 37); Pollard, S. 217 (Tab. 5.10).

${ }^{76}$ Hier verschätzte sich Keynes gewaltig: 1945 betrug das Defizit im Bereich der unsichtbaren Einkünfte $£ 620$ Millionen (1944: 50 Millionen). Vgl. Feinstein, Statistical Tables, T 82 (Tab. 37).

77 CAB 129/1, CP (45) 112, Memorandum Keynes, „Our Overseas Financial Prospects“, 14.8.1945. Bei Kriegsende belief sich die Schuldenlast Großbritanniens auf $£$ 3,55 Milliarden, davon allein 2,7 Milliarden gegenüber anderen Sterling-Ländern. Vgl. Pollard, S. 219 Tab. 5.11. 
staltende Ziel der Vollbeschäftigung zu gewährleisten. Auch der Unterhalt der Streitkräfte drohte nach Keynes' Schätzungen trotz Demobilisierung angesichts der zahlreichen überseeischen Verpflichtungen den Haushalt weiterhin übermäßig zu belasten.

Aufgrund dieses Befundes fühlte sich Keynes zunächst zu einer massiven Kritik der zuständigen Minister berechtigt, die sich seiner Meinung nach wie schon nach dem Ersten Weltkrieg in den Jahren der Kriegszielplanungen zu sehr von politischen Erwägungen hatten leiten lassen. Es entsprach Keynes' Blick auf die außenwirtschaftliche Situation Großbritanniens in diesem Memorandum, daß er an dieser Stelle die Prämissen der britischen Außenpolitik hinterfragte und den Ministern vorhielt, "that our external policies are very far from being adjusted to impending realities“. Vor der anhaltenden Überdehnungstendenz der Außen- und Sicherheitspolitik Londons warnend, fragte er provokativ: "How vividly do Departments and Ministers realise that the gay and successful fashion in which we undertake liabilities all over the world and slop money out to the importunate represents an over-playing of our hand, the possibility of which will come to an end quite suddenly and in the near future unless we obtain a new source of assistance?" 78

Es war keine Frage, daß Keynes im generellen Zusammenhang seiner Ausführungen nicht ausschließlich auf Großbritanniens überseeische Sicherheitsinteressen anspielte, sondern auf die traditionellen Verpflichtungen Londons vor dem Hintergrund der an Bedeutung für den britischen Wiederaufbau vielleicht noch wichtigeren Entwicklungen in Europa, insbesondere hinsichtlich der Deutschlandproblematik, zielte. Denn standen nicht noch zusätzliche Belastungen durch die Besetzung Deutschlands ins Haus? Und warfen die Beschlüsse von Jalta und Potsdam über dessen wirtschaftliche Behandlung nicht ihre rekonstruktionshemmenden Schatten voraus?

Wo in aller Welt, so ließen sich Keynes' Ausführungen in ihrem Kern reduzieren, sollte also das Geld herkommen, und würden die im äußersten Fall zu erwartenden Kredite überhaupt ausreichend Spielraum für die anspruchsvollen außenpolitischen Ziele der Regierung bieten? Vor allem aber: Welche Konsequenzen für die britische Außenpolitik ergaben sich aus der Verwendung der engen Kreditlinie für den sozio-ökonomischen Aufbauprozeß im Inneren? Mit seiner Kritik an den außenpolitischen Grundlagen hatte Keynes bereits angedeutet, daß er eine prinzipielle Reorientierung zugunsten des Wiederaufbaus und der inneren Reformen für notwendig erachtete. Daneben blieb die Frage der Finanzierung der Übergangsphase akut. Keynes konkretisierte daher den bereits im März ventilierten Vorschlag einer Anleihe in den USA. Eine solche Übergangsfinanzierung, soviel war klar, durfte freilich auf keinen Fall die Illusion nähren, daß damit eine fällige Neuorientierung der Außenpolitik überflüssig würde; vielmehr konnte sie bestenfalls eine lebenswichtige Ergänzung hierzu darstellen. Auf der Grundlage der vorliegenden Zahlen ermittelte der Ökonom ein Zahlungsbilanzdefizit bis 1949 in Höhe von rund $£$ 1,7 Milliarden bzw. \$ 5 Milliarden; im Jahr 1949, so kalkulierte er, könne frühestens eine ausgeglichene Zahlungsbilanz erreicht werden, und auch das nur unter gün-

${ }^{78} \mathrm{CAB}$ 129/1, CP (45) 112, Memorandum Keynes, „Our Overseas Financial Prospects“, 14.8.1945. 
stigsten Bedingungen ${ }^{79}$. Dieser Betrag konnte nach Lage der Dinge einzig und allein von den USA kommen und in Keynes' Augen auch nur erfolgreich eingesetzt werden, wenn er die Form einer nichtrückzahlbaren Beihilfe annahm, also in seiner Wirkung nicht durch zusätzliche Zinsbelastungen eingeschränkt würde ${ }^{80}$. Keynes selbst schien sich über den Erfolg eines solchen Anliegens keine sehr großen Illusionen zu machen, zumal neben Umfang und Zinssatz auch die Frage nach den handels- und währungspolitischen Konzessionen eine schwer kalkulierbare Unbekannte darstellte.

Alles in allem also hing die wirtschaftliche und machtpolitische Zukunft Großbritanniens nach Keynes' Analyse davon ab, ob es gelang, die Exportindustrie in entscheidendem Maße auszuweiten, gleichzeitig die außen- und sicherheitspolitischen Verpflichtungen drastisch einzuschränken und Washington davon zu überzeugen, daß nur ein umfangreicher Kredit in Höhe von \$ 5 Milliarden zu den für Großbritannien günstigen Bedingungen das Land in die Lage versetzen werde, rasch auf die Prinzipien von Multilateralität und Konvertibilität umzuschwenken und allgemein die gewünschte Führungsrolle beim Wiederaufbau Europas zu übernehmen. Sollte dieser Kurs scheitern, so warnte er abschließend, gehe das Land einem „finanziellen Dünkirchen“ entgegen: dem erniedrigenden Rückzug aus den bestehenden Verpflichtungen und einem damit einhergehenden massiven Prestigeverlust, dem Absinken auf das Niveau einer zweitklassigen Macht („rather like the present position of France“) sowie Einschränkungen und Rationalisierungen im Inneren, von einer Preisgabe der geplanten Sozialreformen ganz zu schweigen. Vor übertriebenen Hoffnungen wurde also gewarnt. „For the time being Ministers would do well to assume that no arrangement which we can properly accept is yet in sight; and that, until such an arrangement is in sight, we are, with the imminent cessation of Lend-Lease, virtually bankrupt, and the economic basis for the hopes of the public non-existent. "81 Das Kabinett war von dieser schonungslosen Analyse schockiert.

$\begin{array}{lccc}79 & \text { Einige Zahlen (in Mio £): } \\ \text { Keynes' Schätzungen } & \text { Treasury } & \text { Zahlungsbilanz UK } \\ \text { August } 1945 & \text { Oktober } 1945 & \\ 1946 & -950 & -750 & -230 \\ 1947 & -550 & -312 & -381 \\ 1948 & -200 & -125 & 26 \\ 1949 & - & -63 & -1 \\ \text { Gesamt } & -1700 & -1250 & -586\end{array}$

Quellen: CAB 129/1, CP (45) 112, Memorandum Keynes, „Our Overseas Financial Prospects“, 14.8.1945; Keynes an Dalton, 18.10.1945, in: JMK XXIV, S. 555 f., hier: S. 556; Feinstein, Statistical Tables, T 82 (Tab. 37). Die von Keynes in Washington vorgelegten Zahlen sind in Dollar angegeben und auf der Basis des Faktors 4 umgerechnet worden, der auch vom Schatzamt angenommen wurde; die Angabe für 1949 entspricht der Schätzung für 1949/50.

${ }^{80}$ Die Summe von $\$ 5$ Milliarden errechnete sich also aus den direkten Bedürfnissen Großbritanniens im Hinblick auf die breitgefächerte Schuldenlast des Landes und leitete sich unmittelbar aus dem geschätzten Zahlungsbilanzdefizit ab. In seinem Kabinettsmemorandum vom Mai schätzte Keynes, daß die Wirtschaft des Landes etwa \$ 8 Milliarden real benötigte, "to give us real liberty of action and to allow us to offer from the start [...] full multilateralism of trade and exchange", und "to have the full confidence and resources to develop our trade on far-sighted lines and to play our proper part in the world after the war". Diese Summe wurde den Amerikanern in den Verhandlungen dann allerdings nicht mitgeteilt. Vgl. Memorandum Keynes, „Overseas Financial Policy in Stage III“, [Mai 1945], in: JMK XXIV, S. 256-295, hier: S. 276; vgl. dazu Moggridge, Maynard Keynes, S. 784-788.

${ }^{81}$ CAB 129/1, CP (45) 112, Memorandum Keynes, „Our Overseas Financial Prospects“, 14.8.1945. 


\section{Die anglo-amerikanischen Kreditverhandlungen und die Grundlagen der britischen Nachkriegspolitik}

Der weitere Verlauf ist bekannt und materialreich belegt ${ }^{82}$ : Begleitet von einigen Gerüchten im Vorfeld, traf am 21. August 1945 die formelle Ankündigung ein, daß ab sofort alle Lend-Lease-Lieferungen eingestellt würden ${ }^{83}$. Zwei Tage später erläuterte Keynes dem Kernkabinett sowie Vertretern von Foreign Office, Treasury und Board of Trade seine Ziele für die umgehend einzuleitenden Kreditverhandlungen mit den Amerikanern: Autorisierung ausschließlich für eine nichtrückzahlbare Beihilfe; die Höhe des Kredits müsse mindestens \$ 4 Milliarden betragen; Konzessionen in der Frage der Handelspräferenzen seien an die Reduzierung der US-Zölle zu binden; keine Konzessionen in der Währungsfrage, die Teile der für Großbritannien wichtigen Ausweichklauseln des Bretton-Woods-Abkommens rückgängig machen würden ${ }^{84}$. Der am 6. Dezember 1945 unterzeichnete Anleihevertrag sah dagegen vor: einen kommerziellen Kredit in Höhe von \$3,75 Milliarden (zusätzlich weiterer \$650 Millionen "to clean up Lend-Lease“) zu 2\% Zinsen beginnend nach 5 Jahren; die Verpflichtung Großbritanniens - unter Aufgabe des entsprechenden Bretton-Woods-Paragraphen, der eine Übergangsperiode von unbestimmter Länge festgeschrieben hatte -, den Sterling bereits ein Jahr nach Inkrafttreten des Abkommens, also im Juli 1947, konvertibel zu machen.

Angesichts der angeführten Belege war es nun folgerichtig, daß die beiden Verhandlungsdelegationen im Rahmen ihrer Kreditgespräche tatsächlich die Frage der deutschen Reparationen anschnitten. Keynes verdeutlichte seinen amerikanischen Zuhörern dabei, daß der Umfang der Anleihe sich nicht zuletzt aus der Tatsache legitimiere, daß London vernünftigerweise kaum Reparationslieferungen aus Deutschland erwarte. Zwar erhoffe man einige wenige Lieferungen von Nutzholz und Pottasche; zugleich bezweifelte Keynes aber, ob dies - nicht zuletzt wegen des "First charge"-Prinzips - in der britischen Zahlungsbilanz positiv zu Buche schlagen werde. Tatsächlich könne es leicht sein, daß Großbritannien für diese Lieferungen noch nicht einmal die Kosten tragen könne ${ }^{85}$. Nicht ganz in Übereinstimmung mit der Entscheidungsspitze bekräftigte damit Keynes noch einmal, daß „Economic Security“ gegenüber Deutschland im von ihm definierten weiten Sinne des Begriffs in Wechselwirkung stand mit der erfolgreichen Neuordnung des Weltwirtschaftssystems, d. h. letztlich der Übernahme von monetärer Verantwortung durch die USA in einer Weise, die gegenüber Großbritannien großzügige und seinen Kriegslasten angemessene Hilfe gewährte und gleichzeitig den Rahmen für die wirtschaftliche Einbeziehung Deutschlands in den Prozeß des europäischen Wiederaufbaus

82 Vgl. die vorzügliche Edition der britischen Verhandlungsakten in: DBPO I/3 und JMK XXIV. Für eine Analyse der US-Anleihe vgl. Gardner, Sterling-Dollar Diplomacy, S. 188-207; Block, S. 55-68; Cairncross, Years of Recovery, S. 88-120; Moggridge, Maynard Keynes, S. 796-820.

${ }^{83}$ RAFDEL an Air Ministry, 19.8.1945, in: DBPO I/3, S. 54; Note of Meeting on 20 August, 21.8.1945, in: DBPO I/3, S. 55-57; Harrod, Keynes, S. 595; Gardner, Sterling-Dollar Diplomacy, S. 184-187. Zum Hintergrund der Entscheidung vgl. Balfour an Bevin, 22.8.1945, in: DBPO I/3, S. $67 \mathrm{f}$.

${ }^{84}$ Record of a Meeting of Ministers, 23.8.1945, in: DBPO 1/3, S. 72-77. Vgl. die Vorlage Keynes' für die Verhandlungen „Proposals for financial arrangements in the Sterling Area and between the U.S. and the U.K. to follow after Lend-Lease", [4.9.1945], in: DBPO I/1, S. 93-110.

${ }^{85}$ Vgl. U.S.-U.K. Economic Negotiations Finance Committee, Meeting 20.9.1945, in: JMK XXIV, S. 492-498, hier: S. 497. 
bereitstellte. Denn weshalb sonst hätten die amerikanischen Unterhändler das Thema angerissen? Wollten sie nicht in das Terrain Morgenthauscher Argumentation vorstoßen und behaupten, daß die wirtschaftliche Entmachtung Deutschlands sowie Reparationen und die Übernahme wichtiger deutscher Exportmärkte Dringlichkeit und Umfang der Anleihe reduzierten, lag es für Keynes nur in der Logik der Sache, daß eine konstruktive Stabilisierungspolitik Großbritanniens gegenüber Deutschland das Anliegen Londons, günstige amerikanische Kreditbedingungen zu erhalten, unterstrich. Alles andere kam seiner Meinung nach einem Rückfall in die Denkweise von Versailles gleich. Auch im Verlauf der Anleihegespräche sah sich der Nationalökonom folglich gezwungen, deutschlandpolitische Überzeugungsarbeit zu leisten.

Im Zusammenhang dieser Arbeit lassen sich für die britische Außen- und Sicherheitspolitik der Nachkriegszeit aus den Ergebnissen der anglo-amerikanischen Finanzgespräche und dem damit thematisch eng verknüpften Verlauf der interministeriellen Diskussion über die möglichen Auswirkungen der wirtschaftlichen Situation auf die Diplomatie vor allem zwei Schlüsse ziehen. Der statistische Befund, wie er von Keynes in seiner Denkschrift vom August 1945 zuerst ausgebreitet und hinsichtlich der Konsequenzen analysiert wurde, ist allgemein deshalb von großer Bedeutung, weil er an einem Punkt historischer Weichenstellungen den objektiven Ressourcenrahmen beschreibt, innerhalb dessen sich künftig die Außenpolitik Londons bewegen mußte. Er umriß in Form einer realistischen Bestandsaufnahme das Verhältnis zwischen materiellem Status quo und auf der Basis traditioneller Formulierungsansätze entstandenen außenpolitischen Zielvorgaben. Die präformierende Bedeutung der ökonomischen Rahmenbedingungen ist sowohl im Hinblick auf eine von der Labour-Regierung favorisierte Politik des „economic management" als auch vor dem Hintergrund des außen- und sicherheitspolitischen Entscheidungsfindungsprozesses in Whitehall in der Geschichtswissenschaft akzeptiert worden ${ }^{86}$. Dissens besteht allerdings in der Frage, ob die Entscheidungsspitze in London - vor allem in der Übergangszeit, aber auch später - die notwendigen Konsequenzen zog und sich bei der Formulierung ihrer Außenpolitik innerhalb dieses gegebenen Rahmens bewegte oder am Primat der Außenpolitik festhielt und auf der Basis eines „muddle through“ Ad-hoc-Entscheidungen fällte, die im Kern eher von dem Bemühen um Schadensbegrenzung geleitet wurden ${ }^{87}$. In diesem Kontext ist auch die Bedeutung des britisch-amerikanischen Kreditabkommens insbesondere für die künftige Deutschlandpolitik Londons bislang kaum beachtet worden.

Der Abschluß des Anleihevertrages im Dezember 1945 bildete aus dieser Sicht gewissermaßen einen Schlußstein in Keynes' Bemühen, auf den „Lehren von Versailles“ aufbauend den Prozeß der Reorganisation der Weltwirtschaft und die Frage der zukünftigen wirtschaftlichen Behandlung Deutschlands so aufeinander abzustimmen, daß sie sich nicht wie nach dem Ersten Weltkrieg behinderten, sondern den ökonomischen Wiederaufbau Europas förderten und im besonderen den rekonstruktionswirtschaftlichen Interessen Großbritanniens Rechnung trugen. Ein Scheitern würde die Weichen auch für die Deutschlandpolitik Londons verändern, den Handlungsspielraum weiter veren-

${ }^{86}$ Vgl. Carincross, Years of Recovery, S. XI. Zur Außenpolitik vgl. z. B. Foschepoth, Großbritannien, S. 65; Ovendale, S. 3 f.; Peden, Economic Aspects; Pingel, Aufschwung, S. $41 \mathrm{ff}$.; Watt, Hauptprobleme, S. 15-28; Scharf/Schröder, Einleitung, S. 3 ff.; sowie Ebersold, passim.

${ }^{87}$ So auch Meyers, Economic Security, S. 55 f. 
gen und so in entscheidendem Maße die ökonomische und machtpolitische Entwicklung Großbritanniens auch nach der Übergangsperiode negativ präformieren. Der Druck, gegenüber Deutschland einen Stabilisierungskurs zu steuern, der sich kurzfristig für die Übergangszeit an Keynes' reparationspolitischen Überlegungen und langfristig für die Zeit nach Beendigung der Übergangsphase an seinem Konzept eines deutschen Friedensbeitrags orientierte, wurde durch zwei gegenläufige Wirkungskräfte noch verschärft. So sah sich London einerseits gezwungen, mit einem kommerziellen Kredit vorliebzunehmen, zugleich seine im Bretton-Woods-Abkommen verbriefte Garantie einer unbefristeten Übergangszeit aufgeben und sich statt dessen zur Konvertibilität des Pfundes binnen Jahresfrist verpflichten zu müssen; andererseits gelang es nicht, den durch diese Konzessionen entstehenden ökonomischen Druck wenigstens einigermaßen durch eine reziproke Verpflichtung der USA auf eine Reduzierung ihrer Importzölle auszugleichen. Je unbefriedigender deshalb die Kreditverhandlungen für Großbritannien ausfielen und die ökonomischen Rahmenbedingungen verschlechterten, desto enger wurde der Spielraum für Maßnahmen zur wirtschaftlichen Kontrolle Deutschlands. Und je unbefriedigender der Versuch zur Neuordnung des monetären Systems aus Keynes' Sicht blieb, desto schwieriger mußte sich auch das alte Problem einer Kontrolle der ökonomischen Dynamik Deutschlands im Kontext des andauernden wirtschaftlichen und sozialen Transformationsprozesses Großbritanniens gestalten.

Darüber hinaus bleibt festzuhalten, daß die Kassandra-Rufe Keynes' zwar auch in Foreign Office-Kreisen nachhaltigen Eindruck machten und zu einer grundsätzlichen Erörterung über den zukünftigen Gestaltungsrahmen der Außenpolitik führten. Traditionsgebundenheit, gepaart mit der Überzeugung, die vorwiegend organisatorisch-technischen Probleme der britischen Übergangsphase innen- und außenpolitisch lösen zu können, standen einem vollen Verständnis für den Prozeß der wirtschaftlichen und machtpolitischen Strukturveränderungen jedoch im Wege, wenngleich allgemein das Bewußtsein über die ökonomisch begründete Notwendigkeit einzelner Korrekturen geschärft wurde. Diese ambivalente Haltung war schließlich dafür verantwortlich, daß die Beamten zwar auf Keynes' Vorhaltungen mit einer prinzipiellen Anerkennung der wachsenden Bedeutung ökonomischer Faktoren reagierten, aber nur in der Lage waren, auf die Faktizität eingeschränkter Möglichkeiten im Sinne einer „policy of selection“ zu antworten, welche letztlich doch die traditionellen außenpolitischen Interessen Großbritanniens perpetuierte und die Hauptstoßrichtung einer effizienter gestalteten Finanzdiplomatie neben Südosteuropa im Mittleren und Fernen Osten sah. Keynessche Prinzipien in der Deutschlandpolitik hatten sich über das "First charge“-Prinzip hinaus kaum als durchsetzungsfähig erwiesen, und auch seine Vorlagen zur monetären Reform wurden letztlich durch Washington genau jener Elemente beraubt, die aus britischer Sicht den Grund für die Lösung der Übergangsprobleme hätten legen können. Eine „keynesianische Wende“ in der Deutschlandpolitik, die den Anspruch auf eine umfassende Stabilisierungspolitik mit den Erfordernissen der Reformpolitik im Inneren sowie den vertraglichen und weltwirtschaftlichen Rahmenbedingungen eng verzahnt hätte, blieb aus. 
